\title{
Exploring the potential of Electrospray-Orbitrap for stable isotope analysis using nitrate as a model
}

Andreas Hilkert 1,*, John K. Böhlke ${ }^{2}$, Stanley J. Mroczkowski ${ }^{2}$, Kyle L. Fort ${ }^{1}$, Konstantin Aizikov ${ }^{1}$, Xingchen T. Wang ${ }^{3}$, Sebastian H. Kopf ${ }^{4,5}$, Cajetan Neubauer ${ }^{5, *}$

1. Thermo Fisher Scientific (Bremen), Hanna-Kunath Strasse 11, 28199 Bremen, Germany.

2. United States Geological Survey, Reston, Virginia 20192, USA.

3. Department of Earth and Environmental Sciences, Boston College, Chestnut Hill, MA 02467, USA.

4. Department of Geological Sciences, University of Colorado, Boulder, Colorado 80309, USA.

5. Institute of Arctic and Alpine Research, University of Colorado, Boulder, Colorado 80303, USA.

* Corresponding authors: andreas.hilkert@thermofisher.com (A.H.), 123caj@gmail.com (C.N.)

Content:

- Additional description of datasets and data processing.

- Supplemental Table S1: Screening of environmental samples $\left(\mathrm{AgNO}_{3}\right.$ salts; $\left.\mathrm{n}=1\right)$.

- Supplemental Table S2: Sample descriptions and isotopic data (non-Orbitrap).

- References for Table S2.

- Derivation of equations for calculating $\delta$-values from ratios of isotopologs and sensitivity analysis associated with approximating $\delta$-values from isotopolog intensities.

- A separate spreadsheet (.xlsx) with details on delta scale contraction/expansion, memory effects, linearity, and drift.

- A separate spreadsheet (.xlsx) with details on reproducibility, precision, accuracy of isotope measurements in nitrate reference materials for "with M0" experiments. 


\section{Additional description of datasets and data processing}

Isotopic data (non-Orbitrap) shown in the upper part of Table S2 (reference materials, desert salts, fresh groundwater) were used for datasets 1,2 , and 7. Please note, the lab standard $\mathrm{N} 11\left(\mathrm{KNO}_{3}\right)$ was assigned a $\delta^{18} \mathrm{O}$ value of $+26.7 \%$, which is slightly higher than the IRMS value of $+26.3 \%$ o $\left(\delta^{17} \mathrm{O}\right.$ was not reassigned).

Data in the lower part of Table $\mathrm{S} 2$ (reference materials as $\mathrm{AgNO}_{3}$, desert salts as $\mathrm{AgNO}_{3}$, fresh groundwater as $\mathrm{AgNO}_{3}$ ) were used for datasets 3, 4, 5, and 6. N11 (N-15037-Ag) was used as the lab standard in these experiments with its corresponding $\delta$-values.

\section{Flow injection of USGS reference materials ("with M0"; Table 2; FileS2 and File S3)}

Sample sequences were independently repeated on 3 days $(n=3)$. On each day, blocks of 4 consecutive injections of a USGS reference material were bracketed by 4 consecutive injections of

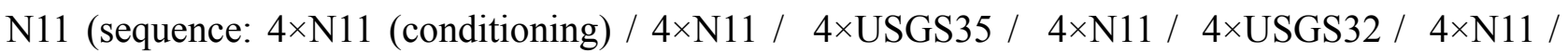
$4 \times$ USGS34 / $4 \times \mathrm{N} 11)$. USGS32 in combination with N11 was used for 2-point calibration of

$\delta^{15} \mathrm{~N}_{\mathrm{sa} / \mathrm{N} 11}$. USGS34 in combination with N11 was used for 2-point calibration of the $\delta^{17} \mathrm{O}_{\text {sa/N11 }}$ and a $0.36 \%$ offset for $\delta^{18} \mathrm{O}_{\mathrm{sa} / \mathrm{N} 11}$. Sample concentration was $50 \mu \mathrm{M}$. No blank correction was applied.

\section{Flow injection of USGS reference materials ("without M0"; Table 2; Figure 2 D-F; File S2)}

Sample sequences were independently repeated on 3 days $(n=3)$. On each day, blocks of 4 consecutive injections of a USGS reference material were bracketed by 4 consecutive injections of

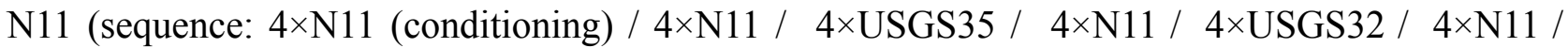
$4 \times$ USGS34 / $4 \times \mathrm{N} 11)$. USGS32 in combination with N11 was used for 2-point calibration of the $\delta\left({ }^{17} \mathrm{O} /{ }^{15} \mathrm{~N}\right)_{\text {sa/N11 }}$ and $\delta\left({ }^{18} \mathrm{O} /{ }^{15} \mathrm{~N}\right)_{\text {sa/N11 }}$ scale. $\delta^{18} \mathrm{O}_{\text {sa/N11 }}$ and $\delta^{17} \mathrm{O}_{\text {sa/N11 }}$ were calculated assuming $\delta^{15} \mathrm{~N}_{\text {sa/N11 }}$ values of samples from Table S2. Sample concentration was $50 \mu \mathrm{M}$. No blank correction was applied.

\section{Flow injection of $\mathrm{AgNO}_{3}$ environmental samples ("with M0"; Table 3)}

Sample sequences were independently repeated on 3 days $(n=3)$. On each day, blocks of 4 consecutive injections of a sample materials were bracketed by 4 consecutive injections of N11 (sequence: $4 \times \mathrm{N} 11$ (conditioning) / $4 \times \mathrm{N} 11 / 4 \times \mathrm{N} 14362 / 4 \times \mathrm{N} 11 / 4 \times \mathrm{N} 17499 / 4 \times \mathrm{N} 11 /$ $4 \times$ N13315 / $4 \times$ N11). An averaged factor of USGS32 in combination with N11 used in Table 2 was applied for 2-point calibration of $\delta^{15} \mathrm{~N}_{\text {sa/N11 }}$ (see above). Sample concentration was $50 \mu$ M. No blank correction was applied.

\section{Flow injection of $\mathrm{AgNO}_{3}$ environmental samples ("without M0"; Table 3)}

Sample sequences were independently repeated on 3 days $(n=3)$. On each day, blocks of 4 consecutive injections of a sample materials were bracketed by 4 consecutive injections of N11 (sequence: $4 \times \mathrm{N} 11$ (conditioning) / $4 \times \mathrm{N} 11 / 4 \times \mathrm{N} 14362 / 4 \times \mathrm{N} 11 / 4 \times \mathrm{N} 17499 / 4 \times \mathrm{N} 11 /$ $4 \times \mathrm{N} 13315$ / $4 \times \mathrm{N} 11)$. The scale correction factors from Table S1 in combination with N11 were applied for 2-point calibration of $\delta\left({ }^{17} \mathrm{O} /{ }^{15} \mathrm{~N}\right)_{\mathrm{sa} / \mathrm{N} 11}$ and $\delta\left({ }^{18} \mathrm{O} /{ }^{15} \mathrm{~N}\right)_{\mathrm{sa} / \mathrm{N} 11}\left(\right.$ see 6.). $\delta^{18} \mathrm{O}_{\text {sa/N11 }}$ and $\delta^{17} \mathrm{O}_{\text {sa/N11 }}$ were calculated assuming $\delta^{15} \mathrm{~N}_{\mathrm{sa} / \mathrm{N} 11}$ values of samples from Table S2. Sample concentration was $50 \mu \mathrm{M}$. No blank correction was applied. 


\section{Flow injection of $\mathrm{AgNO}_{3}$; Screening of environmental samples ("with Mo"; Table S1)}

A single sequence with blocks of 4 consecutive injections of 4 different sample materials $(n=1)$ were bracketed by 4 consecutive injections of N11 (sequence: $4 \times \mathrm{N} 11$ (conditioning) / $4 \times \mathrm{N} 11 /$ N14685, N16854, N13825, N17499 / 4×N11 / N13993, N17008, N13315, N17499 / 4×N11). An averaged factor of USGS32 in combination with N11 used in Table 2 was applied for 2-point calibration of $\delta^{15} \mathrm{~N}_{\mathrm{sa} / \mathrm{N} 11}$ (see 1.). Sample concentration was $50 \mu \mathrm{M}$. No blank correction was applied.

\section{Flow injection of $\mathrm{AgNO}_{3}$; Screening of environmental samples ("without M0"; Table S1)}

A single sequence with blocks of 4 consecutive injections of 4 different sample materials $(n=1)$ were bracketed by 4 consecutive injections of N11 (sequence: $4 \times \mathrm{N} 11$ (conditioning) / $4 \times \mathrm{N} 11 /$ N14685, N16854, N13825, N17499 / 4×N11 / N13993, N17008, N13315, N17499 / 4×N11). Both N17499 injections were used to estimate $\delta$ scale correction factors and were applied in combination with $\mathrm{N} 11$ for 2-point calibration of $\delta\left({ }^{17} \mathrm{O} /{ }^{15} \mathrm{~N}\right)_{\text {sa/N11 }}$ and $\delta\left({ }^{18} \mathrm{O} /{ }^{15} \mathrm{~N}\right)_{\text {sa/N11 }}$ (see above). $\delta^{18} \mathrm{O}_{\text {sa/N11 }}$ and $\delta^{17} \mathrm{O}_{\text {sa/N11 }}$ were calculated assuming $\delta^{15} \mathrm{~N}_{\mathrm{sa} / \mathrm{N} 11}$ values of samples from Table S2. Sample concentration was $50 \mu \mathrm{M}$. No blank correction was applied.

\section{Dual Inlet of $1 \mu M$ environmental samples ("Dilute and shoot"; "with M0"; Table 4)}

Individual Sample / Reference (N11) comparisons with sample / reference bracketing were applied with $10 \mathrm{~min}$ acquisition time each of $\mathrm{Ref} / \mathrm{Sa} / \mathrm{Ref}(30 \mathrm{~min})$ and $\mathrm{Ref} / \mathrm{Sa} / \mathrm{Ref} / \mathrm{Sa} / \mathrm{Ref} / \mathrm{Sa} / \mathrm{Ref}(70 \mathrm{~min})$ methods. After each switch of the Rheodyne valve a 2 min idle time in the data evaluation was applied to avoid any carryover effects from the valve or from the capillary to the ESI source. Data were blank corrected for a blank of $0.18 \mu \mathrm{M}$ with a $\delta^{18} \mathrm{O}$ of $36 \%$ and $\delta^{17} \mathrm{O}$ of $13 \%$. A 1-point calibration with N11 was applied. 


\section{Supplemental Tables}

Table S1. Screening of environmental samples $\left(\operatorname{AgNO}_{3}\right.$ salts; $\left.n=1\right)$.

\begin{tabular}{|c|c|c|c|c|c|c|}
\hline Sample & $\begin{array}{c}\delta^{15} \mathrm{~N}_{\text {Air }}^{\mathrm{a}} \\
\quad E S I\end{array}$ & $\begin{array}{l}\delta^{15} \mathrm{~N}_{\mathrm{Air}} \\
I R M S^{d}\end{array}$ & $\begin{array}{c}\delta^{18} \mathrm{O}_{\text {vSMOW }}{ }^{\mathrm{b}} \\
E S I\end{array}$ & 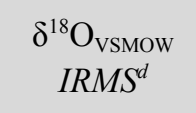 & $\begin{array}{c}\delta^{17} \mathrm{O}_{\text {vSMOw }}{ }^{\mathrm{b}} \\
E S I\end{array}$ & $\begin{array}{c}\delta^{17} \mathrm{O}_{\mathrm{VSMOW}} \\
I R M S^{d}\end{array}$ \\
\hline Death Valley (N14685) & 0.0 & -0.6 & 34.2 & 33.0 & 29.8 & 30.2 \\
\hline Namibia (N16854) & 9.4 & 8.8 & 28.6 & 27.2 & 20.3 & 20.3 \\
\hline UAE Sabkha (N13825) & 11.5 & 10.3 & 33.5 & 31.3 & 25.3 & 25.0 \\
\hline Antarctica (N17499) ${ }^{\mathrm{c}}$ & -15.9 & -16.4 & 80.8 & 80.8 & 73.8 & 73.6 \\
\hline $\begin{array}{l}\text { New Mexico groundwater } \\
(\text { N13993) }\end{array}$ & 6.2 & 6.5 & 2.3 & 0.7 & 1.4 & 0.5 \\
\hline $\begin{array}{l}\text { California groundwater } \\
(\mathrm{N} 17008)\end{array}$ & 7.8 & 7.6 & 5.4 & 3.3 & 3.1 & 3.1 \\
\hline Death Valley (N13315) & 0.7 & 2.9 & 24.2 & 23.1 & 20.0 & 20.1 \\
\hline Antarctica (N17499) ${ }^{\mathrm{c}}$ & -16.2 & -16.4 & 80.9 & 80.8 & 73.2 & 73.6 \\
\hline
\end{tabular}


Table S2. Sample descriptions and isotopic data (non-Orbitrap).

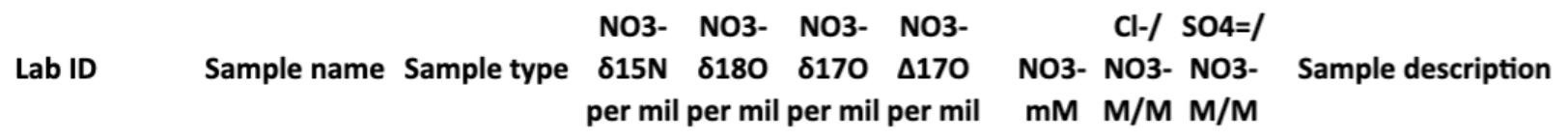

\begin{tabular}{|c|c|c|c|c|c|c|c|c|c|c|}
\hline \multicolumn{11}{|c|}{ Reference materials } \\
\hline USGS32 & USGS32 & KNO3 ref. & 180.0 & 25.3 & 13.0 & -0.2 & $\mathrm{n} / \mathrm{a}$ & $\mathrm{n} / \mathrm{a}$ & $\mathrm{n} / \mathrm{a}$ & Bohlke et al. (1993) \\
\hline USGS34 & USGS34 & KNO3 ref. & -1.8 & -27.8 & -14.8 & -0.3 & $\mathrm{n} / \mathrm{a}$ & $n / a$ & $\mathrm{n} / \mathrm{a}$ & Bohlke et al. (2003) \\
\hline USGS35 & USGS35 & NaNO3 ref. & 2.7 & 56.8 & 51.1 & 21.6 & $\mathrm{n} / \mathrm{a}$ & $\mathrm{n} / \mathrm{a}$ & $\mathrm{n} / \mathrm{a}$ & Bohlke et al. (2003) \\
\hline N11 & N11 & KNO3 ref. & 3.6 & 26.3 & 13.5 & -0.2 & $\mathrm{n} / \mathrm{a}$ & $\mathrm{n} / \mathrm{a}$ & $\mathrm{n} / \mathrm{a}$ & \\
\hline \multicolumn{11}{|c|}{ Desert salts } \\
\hline $\mathrm{N}-14362$ & Atacama & soil leachate & 0.0 & 55.1 & 49.8 & 21.2 & 43.9 & 1.87 & 1.53 & Jackson et al. (2010) \\
\hline $\mathrm{N}-13315$ & Death Valley & soil leachate & 3.5 & 24.2 & 20.7 & 8.1 & 56.6 & $\mathrm{n} / \mathrm{a}$ & $\mathrm{n} / \mathrm{a}$ & Jackson et al. (2010) \\
\hline $\mathrm{N}-14685$ & Death Valley & soil leachate & -0.8 & 34.3 & 30.8 & 13.0 & 23.7 & 13.9 & 2.29 & Jackson et al. (2010) \\
\hline $\mathrm{N}-16854$ & Namibia & brine & 9.1 & 28.6 & 21.0 & 6.1 & 35.8 & 103 & 2.05 & Jackson et al. (2015) \\
\hline N-17499 & Antarctica & soil leachate & -16.2 & 82.5 & 74.5 & 31.6 & 86.4 & $n / a$ & $\mathrm{n} / \mathrm{a}$ & Jackson et al. (2015) \\
\hline $\mathrm{N}-13825$ & UAE sabkha & brine & 10.6 & 32.2 & 25.5 & 8.7 & 173.0 & 40.3 & 0.02 & Jackson et al. (2015) \\
\hline \multicolumn{11}{|c|}{ Fresh groundwater } \\
\hline $\mathrm{N}-13993$ & New Mexico & groundwater & 6.8 & 0.7 & 0.4 & 0.1 & 0.52 & 33.1 & 20.1 & Jackson et al. (2010) \\
\hline N-17008 & California & groundwater & 7.7 & 3.6 & 3.3 & 1.4 & 1.04 & 0.36 & 0.22 & Izbicki et al. (2015) \\
\hline $\mathrm{N}-13819$ & New York & groundwater & 7.3 & 3.5 & 1.8 & 0.0 & 0.50 & 0.63 & 0.50 & Bohlke et al. (2009) \\
\hline $\mathrm{N}-10177$ & Maryland & groundwater & 2.7 & 1.5 & 0.8 & 0.0 & 1.26 & 0.30 & 0.19 & Bohlke and Denver (1995) \\
\hline
\end{tabular}

Reference materials as AgNO3

\begin{tabular}{|c|c|c|c|c|c|c|c|c|c|c|}
\hline N-15038-Ag & USGS34 & AgNO3 ref. & -2.0 & -27.9 & -14.9 & -0.3 & $\mathrm{n} / \mathrm{a}$ & $\mathrm{n} / \mathrm{a}$ & $\mathrm{n} / \mathrm{a}$ & Bohlke et al. (2003) \\
\hline N-15039-Ag & USGS35 & AgNO3 ref. & 2.5 & 56.0 & 50.7 & 21.6 & $\mathrm{n} / \mathrm{a}$ & $\mathrm{n} / \mathrm{a}$ & $\mathrm{n} / \mathrm{a}$ & Bohlke et al. (2003) \\
\hline N-15037-Ag & N11 & AgNO3 ref. & 3.3 & 25.6 & 13.1 & -0.2 & $\mathrm{n} / \mathrm{a}$ & $\mathrm{n} / \mathrm{a}$ & $\mathrm{n} / \mathrm{a}$ & \\
\hline \multicolumn{11}{|c|}{ Desert salts as AgNO3 } \\
\hline N-14362-Ag & Atacama & $\mathrm{AgNO} 3$ & -0.2 & 54.3 & 49.4 & 21.2 & $\mathrm{n} / \mathrm{a}$ & $\mathrm{n} / \mathrm{a}$ & $\mathrm{n} / \mathrm{a}$ & Jackson et al. (2010) \\
\hline N-13315-Ag & Death Valley & AgNO3 & 2.9 & 23.1 & 20.1 & 8.1 & $\mathrm{n} / \mathrm{a}$ & $\mathrm{n} / \mathrm{a}$ & $\mathrm{n} / \mathrm{a}$ & Jackson et al. (2010) \\
\hline $\mathrm{N}-14685-\mathrm{Ag}$ & Death Valley & $\mathrm{AgNO} 3$ & -0.6 & 33.0 & 30.2 & 13.0 & $\mathrm{n} / \mathrm{a}$ & $\mathrm{n} / \mathrm{a}$ & $\mathrm{n} / \mathrm{a}$ & Jackson et al. (2010) \\
\hline $\mathrm{N}-16854-\mathrm{Ag}$ & Namibia & $\mathrm{AgNO} 3$ & 8.8 & 27.2 & 20.3 & 6.1 & $\mathrm{n} / \mathrm{a}$ & $\mathrm{n} / \mathrm{a}$ & $\mathrm{n} / \mathrm{a}$ & Jackson et al. (2015) \\
\hline $\mathrm{N}-17499-\mathrm{Ag}$ & Antarctica & AgNO3 & -16.4 & 80.8 & 73.6 & 31.6 & $\mathrm{n} / \mathrm{a}$ & $\mathrm{n} / \mathrm{a}$ & $\mathrm{n} / \mathrm{a}$ & Jackson et al. (2015) \\
\hline N-13825-Ag & UAE sabkha & $\mathrm{AgNO} 3$ & 10.3 & 31.3 & 25.0 & 8.7 & $\mathrm{n} / \mathrm{a}$ & $\mathrm{n} / \mathrm{a}$ & $\mathrm{n} / \mathrm{a}$ & Jackson et al. (2015) \\
\hline \multicolumn{11}{|c|}{ Fresh groundwater as AgNO3 } \\
\hline N-13993-Ag & New Mexico & $\mathrm{AgNO} 3$ & 6.5 & 0.7 & 0.5 & 0.1 & $\mathrm{n} / \mathrm{a}$ & $\mathrm{n} / \mathrm{a}$ & $\mathrm{n} / \mathrm{a}$ & Jackson et al. (2010) \\
\hline N-17008-Ag & California & AgNO3 & 7.6 & 3.3 & 3.1 & 1.4 & $\mathrm{n} / \mathrm{a}$ & $\mathrm{n} / \mathrm{a}$ & $\mathrm{n} / \mathrm{a}$ & Izbicki et al. (2015) \\
\hline
\end{tabular}

\section{Sources of isotope data in Table S2:}

$\delta^{15} \mathrm{~N}$ reference values $( \pm 0.2)$ were compiled from Bohlke and Coplen $(1995)^{1}$ and Bohlke et al. $(2003)^{2}$, normalized to $-1.8 \%$ for USGS34 and $+180 \%$ for USGS32 (bold font).

$\delta^{15} \mathrm{~N}$ values $( \pm 0.3)$ for all environmental samples and all $\mathrm{AgNO}_{3}$ samples were determined using the bacterial reduction method with $P$. aureofaciens (Casciotti et al., 2002), normalized to $-1.8 \%$ for USGS34 and $+180 \%$ for USGS32, and then adjusted to account for excess ${ }^{17} \mathrm{O}$ by subtracting $1.1 \mathrm{x}$ $\Delta^{17} \mathrm{O} / 21.6$ (Bohlke et al., 2003). ${ }^{2}$

$\delta^{18} \mathrm{O}$ reference values $( \pm 0.2-0.3)$ from Bohlke et al. $(2003)^{2}$ were renormalized to be consistent with USGS34 and USGS35 data from Brand et al. (2009) ${ }^{4}$ (bold font).

$\delta^{18} \mathrm{O}$ values $( \pm 0.4)$ for all environmental samples and all $\mathrm{AgNO}_{3}$ samples were determined using the 
bacterial reduction method with $P$. aureofaciens (Casciotti et al., 2002) ${ }^{3}$, normalized to -27.8 for USGS34 and +56.8 for USGS35 (bold font).

$\delta^{17} \mathrm{O}$ values for all samples were calculated from measured $\delta^{18} \mathrm{O}$ and $\Delta^{17} \mathrm{O}$ values using the relation: $\delta^{17} \mathrm{O}=\Delta^{17} \mathrm{O}+0.52 \times \delta^{18} \mathrm{O}$. Please note, this is the simplest definition of $\Delta^{17} \mathrm{O}$ and can introduce additional uncertainties.

$\Delta^{17} \mathrm{O}$ reference values $( \pm 0.1-0.2)$ were compiled from Michalski et al. $(2002)^{5}$ and Bohlke et al. $(2003)^{2}$, using the definition $\Delta^{17} \mathrm{O}=\delta^{17} \mathrm{O}-0.52 \times \delta^{18} \mathrm{O}$ (bold font); USGS32 was assumed to be similar to other reagents.

$\Delta^{17} \mathrm{O}$ values $( \pm 0.2)$ for environmental $\mathrm{AgNO}_{3}$ samples were determined on oxygen gas liberated by decomposition of $\mathrm{AgNO}_{3}$ (Jackson et al., 2010; modified from Michalski et al., 2002) ${ }^{5,6} ; \Delta^{17} \mathrm{O}$ values were assumed to be the same in $\mathrm{AgNO}_{3}$ and corresponding aqueous samples; New York and Maryland groundwater samples (no $\mathrm{AgNO}_{3}$ ) were assumed to have $\Delta^{17} \mathrm{O}=0$ (biogenic nitrate).

Overall uncertainties could be larger in some cases than the estimated reproducibilities (1-sigma) listed above.

\section{References for Table S2}

(1) Böhlke, J. K.; Coplen, T. B. Interlaboratory Comparison of Reference Materials for Nitrogen-Isotope-Ratio Measurements; IAEA-TECDOC-825; IAEA, 1995.

(2) Böhlke, J. K.; Mroczkowski, S. J.; Coplen, T. B. Oxygen Isotopes in Nitrate: New Reference Materials for ${ }^{18} \mathrm{O}:{ }^{17} \mathrm{O}:{ }^{16} \mathrm{O}$ Measurements and Observations on Nitrate-Water Equilibration. Rapid Commun. Mass Spectrom. 2003, 17 (16), 1835-1846.

(3) Casciotti, K. L.; Sigman, D. M.; Hastings, M. G.; Böhlke, J. K.; Hilkert, A. Measurement of the Oxygen Isotopic Composition of Nitrate in Seawater and Freshwater Using the Denitrifier Method. Anal. Chem. 2002, 74 (19), 4905-4912.

(4) Brand, W. A.; Coplen, T. B.; Aerts-Bijma, A. T.; Böhlke, J. K.; Gehre, M.; Geilmann, H.; Gröning, M.; Jansen, H. G.; Meijer, H. A. J.; Mroczkowski, S. J.; Others. Comprehensive Inter-Laboratory Calibration of Reference Materials for $\delta^{18} \mathrm{O}$ versus VSMOW Using Various On-Line High-Temperature Conversion Techniques. Rapid Communications in Mass Spectrometry 2009, 23 (7), 999-1019.

(5) Michalski, G.; Savarino, J.; Böhlke, J. K.; Thiemens, M. Determination of the Total Oxygen Isotopic Composition of Nitrate and the Calibration of a $\Delta^{17} \mathrm{O}$ Nitrate Reference Material. Anal. Chem. 2002, 74 (19), 4989-4993.

(6) Jackson, W. A.; Böhlke, J. K.; Gu, B.; Hatzinger, P. B.; Sturchio, N. C. Isotopic Composition and Origin of Indigenous Natural Perchlorate and Co-Occurring Nitrate in the Southwestern United States. Environ. Sci. Technol. 2010, 44 (13), 4869-4876.

(7) Izbicki, J. A.; Teague, N. F.; Hatzinger, P. B.; Böhlke, J. K.; Sturchio, N. C. Groundwater Movement, Recharge, and Perchlorate Occurrence in a Faulted Alluvial Aquifer in California (USA). Hydrogeol. J. 2015, 23 (3), 467-491.

(8) Böhlke, J. K.; Denver, J. M. Combined Use of Groundwater Dating, Chemical, and Isotopic Analyses to Resolve the History and Fate of Nitrate Contamination in Two Agricultural Watersheds, Atlantic Coastal Plain, Maryland. Water Resour. Res. 1995, 31 (9), 2319-2339. 
(9) Jackson, W. A.; Böhlke, J. K.; Andraski, B. J.; Fahlquist, L.; Bexfield, L.; Eckardt, F. D.; Gates, J. B.; Davila, A. F.; McKay, C. P.; Rao, B.; Sevanthi, R.; Rajagopalan, S.; Estrada, N.; Sturchio, N.; Hatzinger, P. B.; Anderson, T. A.; Orris, G.; Betancourt, J.; Stonestrom, D.; Latorre, C.; Li, Y.; Harvey, G. J. Global Patterns and Environmental Controls of Perchlorate and Nitrate Co-Occurrence in Arid and Semi-Arid Environments. Geochim. Cosmochim. Acta 2015, 164, $502-522$. 


\section{Equations for calculating delta values from ratios of isotopologs}

\section{Contents}

Ions and their stochastic makeup $\quad$ S9

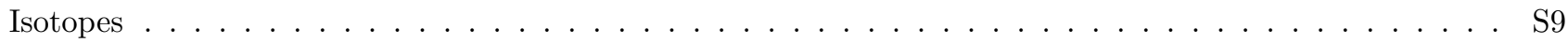

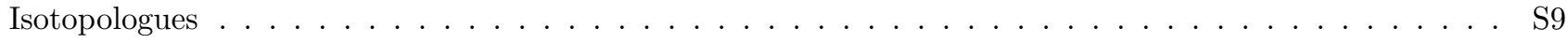

$\delta$-values from analysis with base peak ("with M0") $\mathrm{S12}$

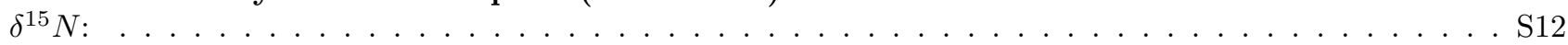

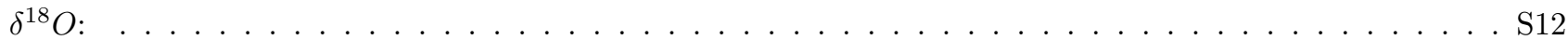

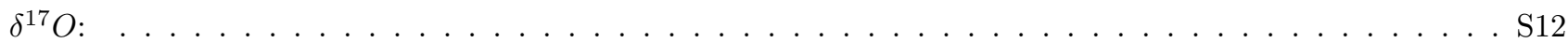

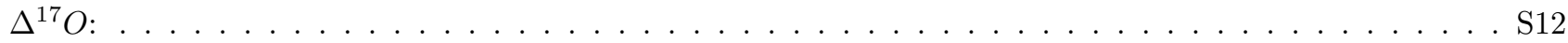

$\delta$-values from analysis without base peak ("without M0") $\quad \mathrm{S12}$

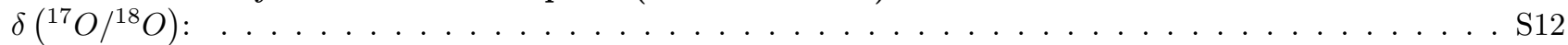

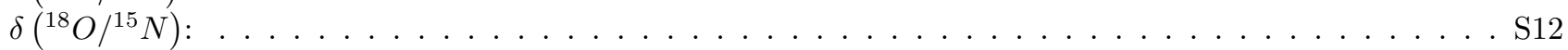

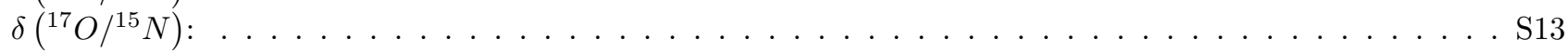

$\begin{array}{lr}\text { Possible } \delta \text {-value calculations } & \mathbf{S 1 3}\end{array}$

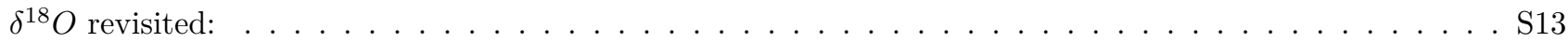

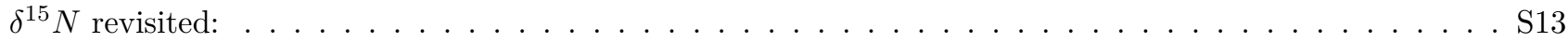

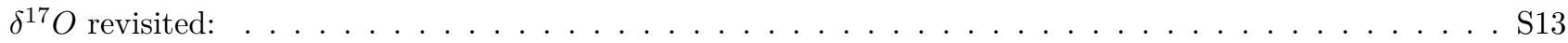

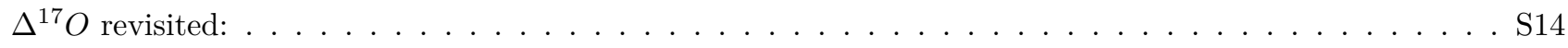

Clumped isotopes $\quad$ S15

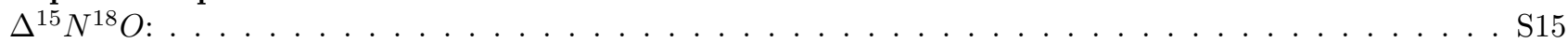

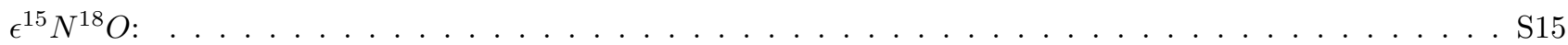

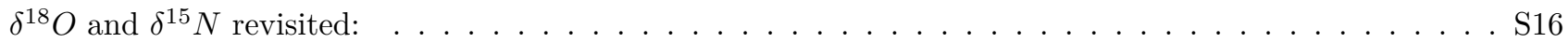

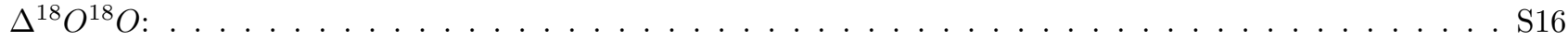

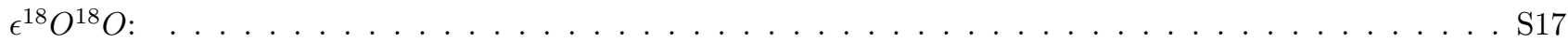

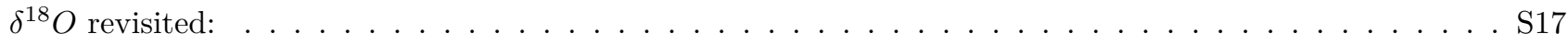

$\begin{array}{lr}\text { Summary for } \delta^{15} N, \delta^{18} O, \delta^{17} O, \Delta^{17} O & \text { S18 }\end{array}$

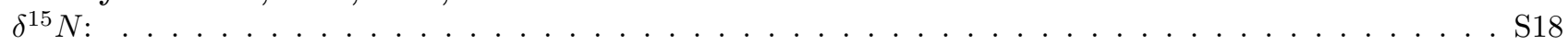

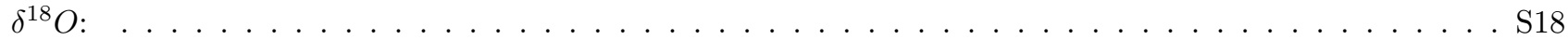

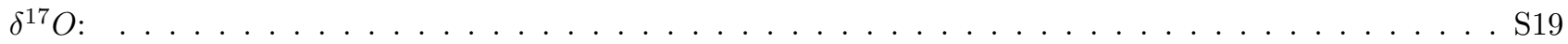

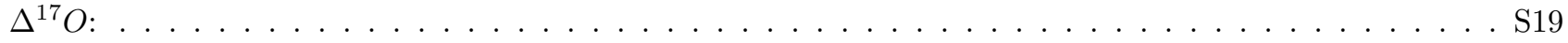

$\begin{array}{lr}\text { Potential error from isotopologue ratio calculations } & \text { S19 }\end{array}$

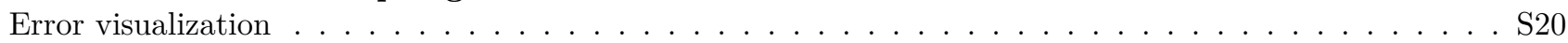




\section{Ions and their stochastic makeup}

Exact masses are based on NIST data base at https://www.nist.gov/pml/atomic-weights-and-isotopiccompositions-relative-atomic-masses. The isotopic abundances are best measurement abundances of the international referencdes standards Air N2 and VSMOW (sources: https://www.ciaaw.org/; Meija, J. and others, 2016, Isotopic compositions of the elements 2013 (IUPAC Technical Report). Pure and Applied Chemistry, v. 88(3), p. 293-306, https://doi.org/10.1515/pac-2015-0503; Coplen, T.B., and others, 2002, Isotope-abundance variations of selected elements. Pure and Applied Chemistry, v. 74, p. 1987-2017, https://doi.org/10.1351/pac200274101987).

\section{Isotopes}

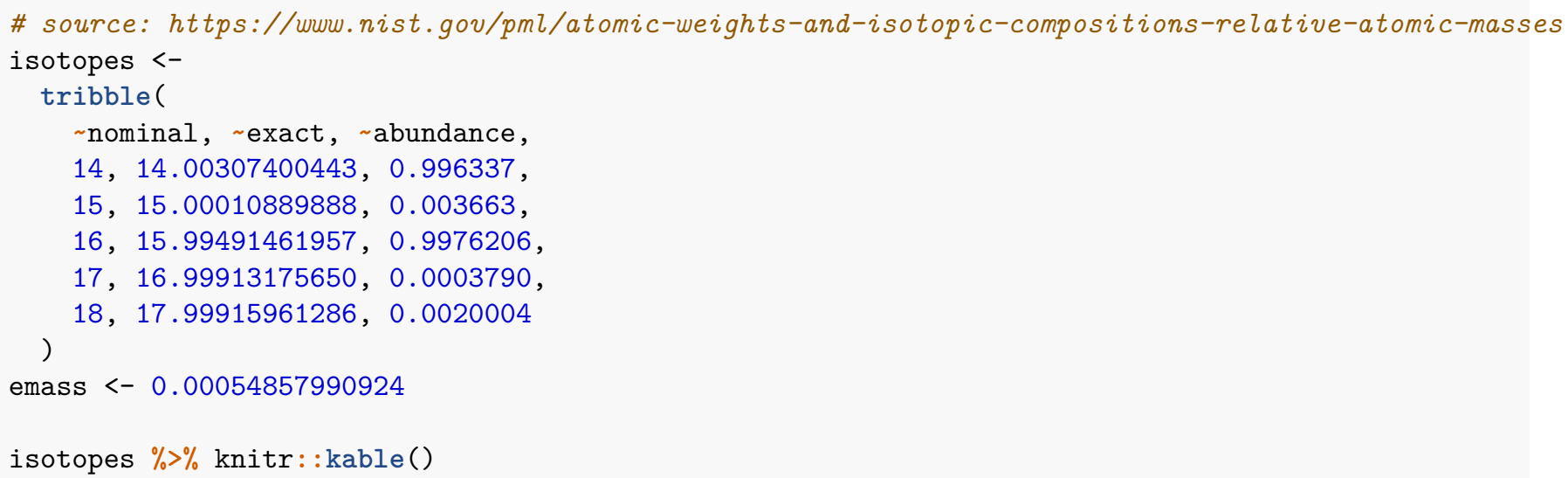

\begin{tabular}{rrr}
\hline nominal & exact & abundance \\
\hline 14 & 14.00307 & 0.9963370 \\
15 & 15.00011 & 0.0036630 \\
16 & 15.99491 & 0.9976206 \\
17 & 16.99913 & 0.0003790 \\
18 & 17.99916 & 0.0020004 \\
\hline
\end{tabular}

\section{Isotopologues}

Note: abundances of the isotopologues are stochastic (i.e. assuming no clumping or anticlumping).

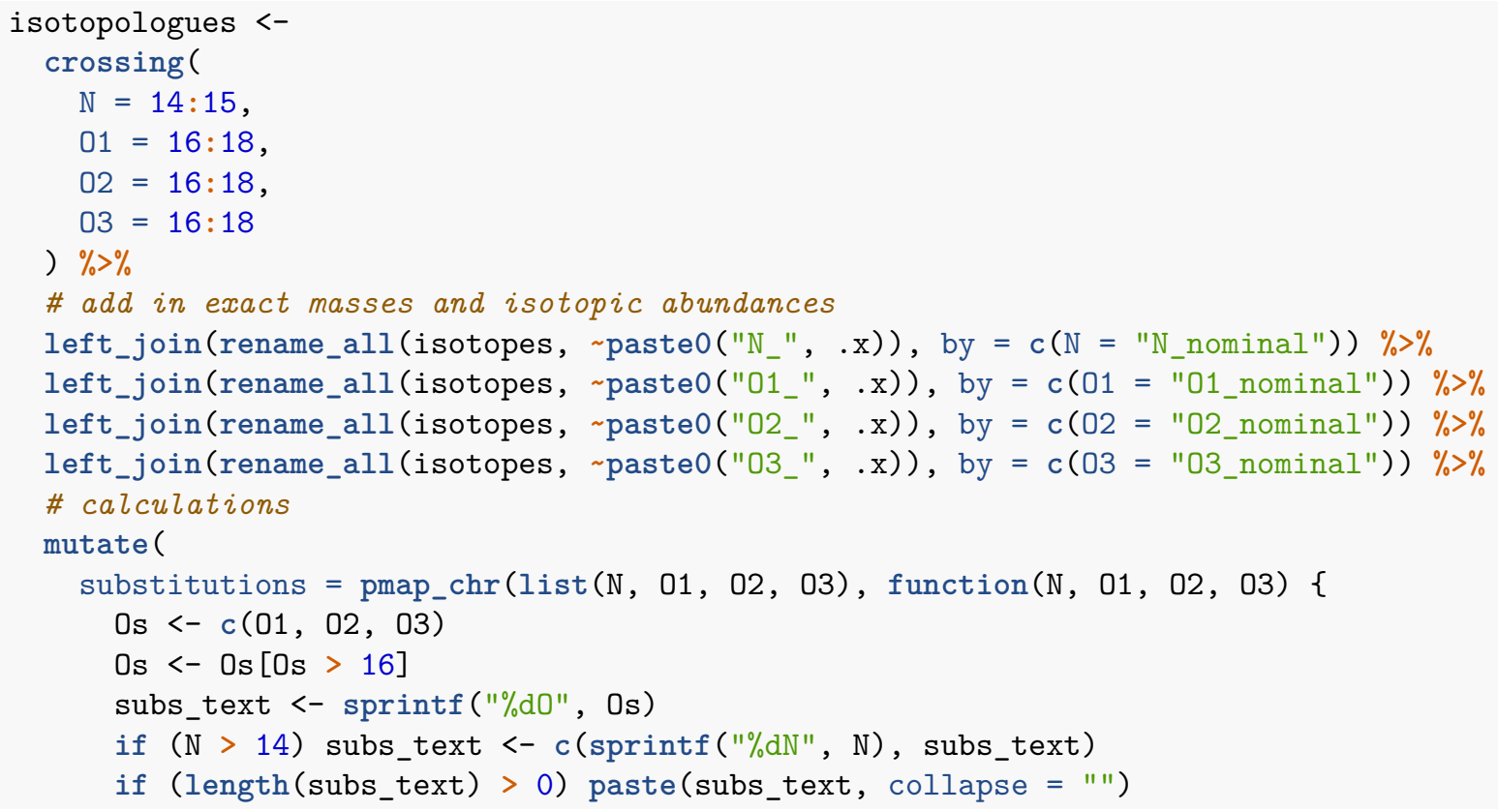




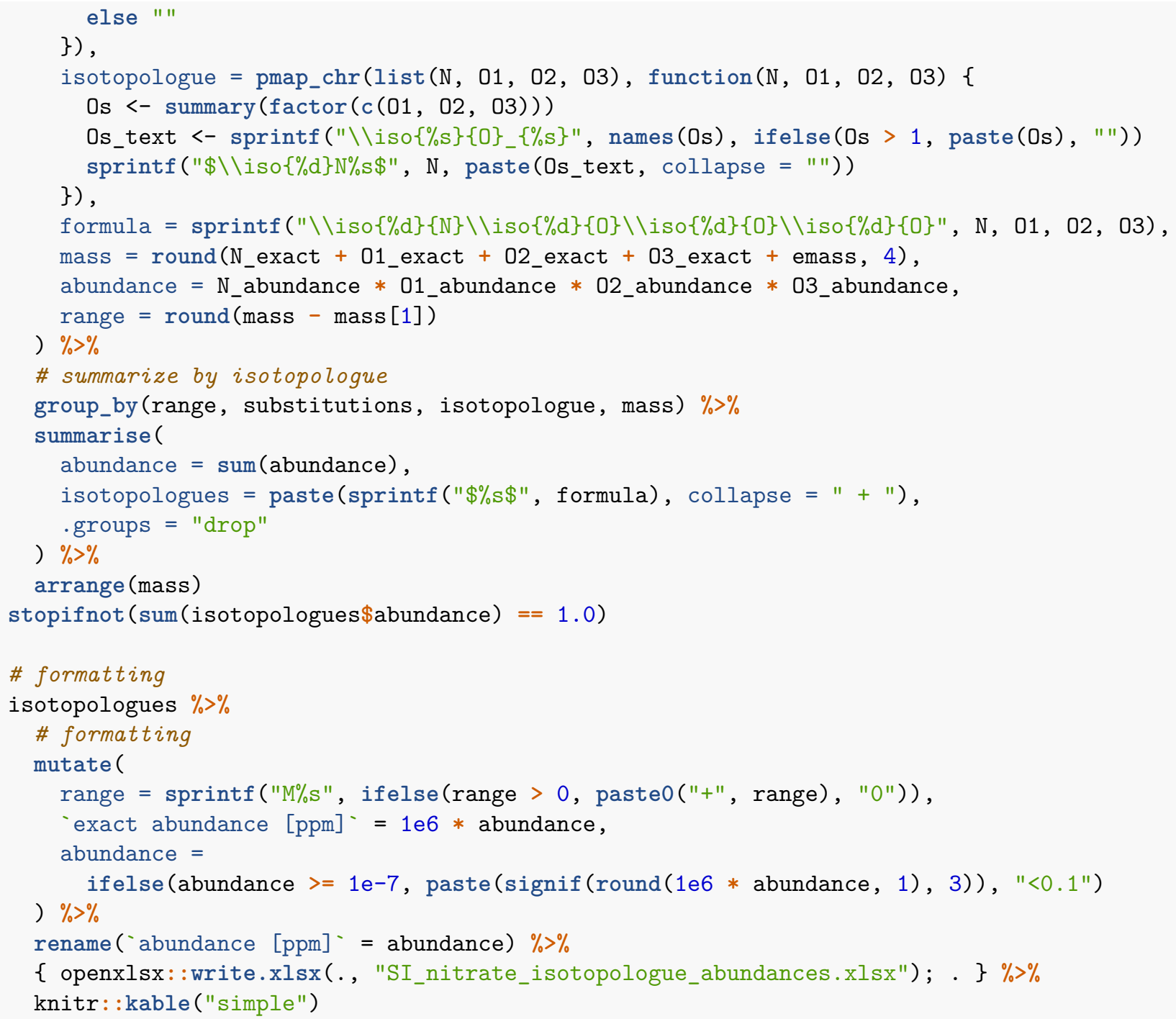

\begin{tabular}{|c|c|c|c|c|c|}
\hline range & substitutions & isotopologue & mass & abundance [ppm] & isotopologues \\
\hline M0 & & ${ }^{14} \mathrm{~N}^{16} \mathrm{O}_{3}$ & 61.9884 & 989000 & ${ }^{14} \mathrm{~N}^{16} \mathrm{O}^{16} \mathrm{O}^{16} \mathrm{O}$ \\
\hline$M+1$ & $15 \mathrm{~N}$ & ${ }^{15} \mathrm{~N}^{16} \mathrm{O}_{3}$ & 62.9854 & 3640 & ${ }^{15} \mathrm{~N}^{16} \mathrm{O}^{16} \mathrm{O}^{16} \mathrm{O}$ \\
\hline $\mathrm{M}+1$ & $17 \mathrm{O}$ & ${ }^{14} \mathrm{~N}^{16} \mathrm{O}_{2}{ }^{17} \mathrm{O}$ & 62.9926 & 1130 & ${ }^{14} \mathrm{~N}^{16} \mathrm{O}^{16} \mathrm{O}^{17} \mathrm{O}+{ }^{14} \mathrm{~N}^{16} \mathrm{O}^{17} \mathrm{O}^{16} \mathrm{O}+{ }^{14} \mathrm{~N}^{17} \mathrm{O}^{16} \mathrm{O}^{16} \mathrm{O}$ \\
\hline $\mathrm{M}+2$ & $15 \mathrm{~N} 17 \mathrm{O}$ & ${ }^{15} \mathrm{~N}^{16} \mathrm{O}_{2}{ }^{17} \mathrm{O}$ & 63.9896 & 4.1 & ${ }^{15} \mathrm{~N}^{16} \mathrm{O}^{16} \mathrm{O}^{17} \mathrm{O}+{ }^{15} \mathrm{~N}^{16} \mathrm{O}^{17} \mathrm{O}^{16} \mathrm{O}+{ }^{15} \mathrm{~N}^{17} \mathrm{O}^{16} \mathrm{O}^{16} \mathrm{O}$ \\
\hline $\mathrm{M}+2$ & $18 \mathrm{O}$ & ${ }^{14} \mathrm{~N}^{16} \mathrm{O}_{2}{ }^{18} \mathrm{O}$ & 63.9926 & 5950 & ${ }^{14} \mathrm{~N}^{16} \mathrm{O}^{16} \mathrm{O}^{18} \mathrm{O}+{ }^{14} \mathrm{~N}^{16} \mathrm{O}^{18} \mathrm{O}^{16} \mathrm{O}+{ }^{14} \mathrm{~N}^{18} \mathrm{O}^{16} \mathrm{O}^{16} \mathrm{O}$ \\
\hline $\mathrm{M}+2$ & $17 \mathrm{O} 17 \mathrm{O}$ & ${ }^{14} \mathrm{~N}^{16} \mathrm{O}^{17} \mathrm{O}_{2}$ & 63.9968 & 0.4 & ${ }^{14} \mathrm{~N}^{16} \mathrm{O}^{17} \mathrm{O}^{17} \mathrm{O}+{ }^{14} \mathrm{~N}^{17} \mathrm{O}^{16} \mathrm{O}^{17} \mathrm{O}+{ }^{14} \mathrm{~N}^{17} \mathrm{O}^{17} \mathrm{O}^{16} \mathrm{O}$ \\
\hline $\mathrm{M}+3$ & $15 \mathrm{~N} 18 \mathrm{O}$ & ${ }^{15} \mathrm{~N}^{16} \mathrm{O}_{2}{ }^{18} \mathrm{O}$ & 64.9896 & 21.9 & ${ }^{15} \mathrm{~N}^{16} \mathrm{O}^{16} \mathrm{O}^{18} \mathrm{O}+{ }^{15} \mathrm{~N}^{16} \mathrm{O}^{18} \mathrm{O}^{16} \mathrm{O}+{ }^{15} \mathrm{~N}^{18} \mathrm{O}^{16} \mathrm{O}^{16} \mathrm{O}$ \\
\hline $\mathrm{M}+3$ & $15 \mathrm{~N} 17 \mathrm{O} 17 \mathrm{O}$ & ${ }^{15} \mathrm{~N}^{16} \mathrm{O}^{17} \mathrm{O}_{2}$ & 64.9938 & $<0.1$ & ${ }^{15} \mathrm{~N}^{16} \mathrm{O}^{17} \mathrm{O}^{17} \mathrm{O}+{ }^{15} \mathrm{~N}^{17} \mathrm{O}^{16} \mathrm{O}^{17} \mathrm{O}+{ }^{15} \mathrm{~N}^{17} \mathrm{O}^{17} \mathrm{O}^{16} \mathrm{O}$ \\
\hline $\mathrm{M}+3$ & $17 \mathrm{O} 18 \mathrm{O}$ & ${ }^{14} \mathrm{~N}^{16} \mathrm{O}^{17} \mathrm{O}^{18} \mathrm{O}$ & 64.9968 & 2.3 & ${ }^{14} \mathrm{~N}^{16} \mathrm{O}^{17} \mathrm{O}^{18} \mathrm{O}+{ }^{14} \mathrm{~N}^{17} \mathrm{O}^{16} \mathrm{O}^{18} \mathrm{O}+{ }^{14} \mathrm{~N}^{17} \mathrm{O}^{18} \mathrm{O}^{16} \mathrm{O}$ \\
\hline $\mathrm{M}+3$ & $18 \mathrm{O} 17 \mathrm{O}$ & ${ }^{14} \mathrm{~N}^{16} \mathrm{O}^{17} \mathrm{O}^{18} \mathrm{O}$ & 64.9968 & 2.3 & ${ }^{14} \mathrm{~N}^{16} \mathrm{O}^{18} \mathrm{O}^{17} \mathrm{O}+{ }^{14} \mathrm{~N}^{18} \mathrm{O}^{16} \mathrm{O}^{17} \mathrm{O}+{ }^{14} \mathrm{~N}^{18} \mathrm{O}^{17} \mathrm{O}^{16} \mathrm{O}$ \\
\hline $\mathrm{M}+3$ & $17 \mathrm{O} 17 \mathrm{O} 17 \mathrm{O}$ & ${ }^{14} \mathrm{~N}^{17} \mathrm{O}_{3}$ & 65.0010 & $<0.1$ & ${ }^{14} \mathrm{~N}^{17} \mathrm{O}^{17} \mathrm{O}^{17} \mathrm{O}$ \\
\hline $\mathrm{M}+4$ & $15 \mathrm{~N} 17 \mathrm{O} 18 \mathrm{O}$ & ${ }^{15} \mathrm{~N}^{16} \mathrm{O}^{17} \mathrm{O}^{18} \mathrm{O}$ & 65.9939 & $<0.1$ & ${ }^{15} \mathrm{~N}^{16} \mathrm{O}^{17} \mathrm{O}^{18} \mathrm{O}+{ }^{15} \mathrm{~N}^{17} \mathrm{O}^{16} \mathrm{O}^{18} \mathrm{O}+{ }^{15} \mathrm{~N}^{17} \mathrm{O}^{18} \mathrm{O}^{16} \mathrm{O}$ \\
\hline $\mathrm{M}+4$ & $15 \mathrm{~N} 18 \mathrm{O} 17 \mathrm{O}$ & ${ }^{15} \mathrm{~N}^{16} \mathrm{O}^{17} \mathrm{O}^{18} \mathrm{O}$ & 65.9939 & $<0.1$ & ${ }^{15} \mathrm{~N}^{16} \mathrm{O}^{18} \mathrm{O}^{17} \mathrm{O}+{ }^{15} \mathrm{~N}^{18} \mathrm{O}^{16} \mathrm{O}^{17} \mathrm{O}+{ }^{15} \mathrm{~N}^{18} \mathrm{O}^{17} \mathrm{O}^{16} \mathrm{O}$ \\
\hline $\mathrm{M}+4$ & $18 \mathrm{O} 18 \mathrm{O}$ & ${ }^{14} \mathrm{~N}^{16} \mathrm{O}^{18} \mathrm{O}_{2}$ & 65.9969 & 11.9 & ${ }^{14} \mathrm{~N}^{16} \mathrm{O}^{18} \mathrm{O}^{18} \mathrm{O}+{ }^{14} \mathrm{~N}^{18} \mathrm{O}^{16} \mathrm{O}^{18} \mathrm{O}+{ }^{14} \mathrm{~N}^{18} \mathrm{O}^{18} \mathrm{O}^{16} \mathrm{O}$ \\
\hline $\mathrm{M}+4$ & $15 \mathrm{~N} 17 \mathrm{O} 17 \mathrm{O} 17 \mathrm{O}$ & ${ }^{15} \mathrm{~N}^{17} \mathrm{O}_{3}$ & 65.9981 & $<0.1$ & ${ }^{15} \mathrm{~N}^{17} \mathrm{O}^{17} \mathrm{O}^{17} \mathrm{O}$ \\
\hline $\mathrm{M}+4$ & $17 \mathrm{O} 17 \mathrm{O} 18 \mathrm{O}$ & ${ }^{14} \mathrm{~N}^{17} \mathrm{O}_{2}{ }^{18} \mathrm{O}$ & 66.0010 & $<0.1$ & ${ }^{14} \mathrm{~N}^{17} \mathrm{O}^{17} \mathrm{O}^{18} \mathrm{O}$ \\
\hline $\mathrm{M}+4$ & $17 \mathrm{O} 18 \mathrm{O} 17 \mathrm{O}$ & ${ }^{14} \mathrm{~N}^{17} \mathrm{O}_{2}{ }^{18} \mathrm{O}$ & 66.0010 & $<0.1$ & ${ }^{14} \mathrm{~N}^{17} \mathrm{O}^{18} \mathrm{O}^{17} \mathrm{O}$ \\
\hline $\mathrm{M}+4$ & 180170170 & ${ }^{14} \mathrm{~N}^{17} \mathrm{O}_{2}{ }^{18} \mathrm{O}$ & 66.0010 & $<0.1$ & ${ }^{14} \mathrm{~N}^{18} \mathrm{O}^{17} \mathrm{O}^{17} \mathrm{O}$ \\
\hline $\mathrm{M}+5$ & $15 \mathrm{~N} 18 \mathrm{O} 18 \mathrm{O}$ & ${ }^{15} \mathrm{~N}^{16} \mathrm{O}^{18} \mathrm{O}_{2}$ & 66.9939 & $<0.1$ & ${ }^{15} \mathrm{~N}^{16} \mathrm{O}^{18} \mathrm{O}^{18} \mathrm{O}+{ }^{15} \mathrm{~N}^{18} \mathrm{O}^{16} \mathrm{O}^{18} \mathrm{O}+{ }^{15} \mathrm{~N}^{18} \mathrm{O}^{18} \mathrm{O}^{16} \mathrm{O}$ \\
\hline$M+5$ & $15 \mathrm{~N} 17 \mathrm{O} 17 \mathrm{O} 18 \mathrm{O}$ & ${ }^{15} \mathrm{~N}^{17} \mathrm{O}_{2}{ }^{18} \mathrm{O}$ & 66.9981 & $<0.1$ & ${ }^{15} \mathrm{~N}^{17} \mathrm{O}^{17} \mathrm{O}^{18} \mathrm{O}$ \\
\hline
\end{tabular}




\begin{tabular}{lllrll}
\hline range & substitutions & isotopologue & mass & abundance $[\mathrm{ppm}]$ & isotopologues \\
\hline $\mathrm{M}+5$ & $15 \mathrm{~N} 17 \mathrm{O} 18 \mathrm{O} 17 \mathrm{O}$ & ${ }^{15} \mathrm{~N}^{17} \mathrm{O}_{2}{ }^{18} \mathrm{O}$ & 66.9981 & $<0.1$ & ${ }^{15} \mathrm{~N}^{17} \mathrm{O}^{18} \mathrm{O}^{17} \mathrm{O}$ \\
$\mathrm{M}+5$ & $15 \mathrm{~N} 18 \mathrm{O} 17 \mathrm{O} 17 \mathrm{O}$ & ${ }^{15} \mathrm{~N}^{17} \mathrm{O}_{2}^{18} \mathrm{O}$ & 66.9981 & $<0.1$ & ${ }^{15} \mathrm{~N}^{18} \mathrm{O}^{17} \mathrm{O}^{17} \mathrm{O}$ \\
$\mathrm{M}+5$ & $17 \mathrm{O} 18 \mathrm{O} 18 \mathrm{O}$ & ${ }^{14} \mathrm{~N}^{17} \mathrm{O}^{18} \mathrm{O}_{2}$ & 67.0011 & $<0.1$ & ${ }^{14} \mathrm{~N}^{17} \mathrm{O}^{18} \mathrm{O}^{18} \mathrm{O}$ \\
$\mathrm{M}+5$ & $18 \mathrm{O} 17 \mathrm{O} 18 \mathrm{O}$ & ${ }^{14} \mathrm{~N}^{17} \mathrm{O}^{18} \mathrm{O}_{2}$ & 67.0011 & $<0.1$ & ${ }^{14} \mathrm{~N}^{18} \mathrm{O}^{17} \mathrm{O}^{18} \mathrm{O}$ \\
$\mathrm{M}+5$ & $18 \mathrm{O} 18 \mathrm{O} 17 \mathrm{O}$ & ${ }^{14} \mathrm{~N}^{17} \mathrm{O}^{18} \mathrm{O}_{2}$ & 67.0011 & $<0.1$ & ${ }^{15} \mathrm{~N}^{17} \mathrm{O}^{18} \mathrm{O}^{18} \mathrm{O}$ \\
$\mathrm{M}+6$ & $15 \mathrm{~N} 17 \mathrm{O} 18 \mathrm{O} 18 \mathrm{O}$ & ${ }^{15} \mathrm{~N}^{17} \mathrm{O}^{18} \mathrm{O}_{2}$ & 67.9981 & $<0.1$ & ${ }^{15} \mathrm{~N}^{18} \mathrm{O}^{17} \mathrm{O}^{18} \mathrm{O}$ \\
$\mathrm{M}+6$ & $15 \mathrm{~N} 18 \mathrm{O} 17 \mathrm{O} 18 \mathrm{O}$ & ${ }^{15} \mathrm{~N}^{17} \mathrm{O}^{18} \mathrm{O}_{2}$ & 67.9981 & $<0.1$ & ${ }^{15} \mathrm{~N}^{18} \mathrm{O}^{18} \mathrm{O}^{17} \mathrm{O}$ \\
$\mathrm{M}+6$ & $15 \mathrm{~N} 18 \mathrm{O} 18 \mathrm{O} 17 \mathrm{O}$ & ${ }^{15} \mathrm{~N}^{17} \mathrm{O}^{18} \mathrm{O}_{2}$ & 67.9981 & $<0.1$ & ${ }^{14} \mathrm{~N}^{18} \mathrm{O}^{18} \mathrm{O}^{18} \mathrm{O}$ \\
$\mathrm{M}+6$ & $18 \mathrm{O} 18 \mathrm{O} 18 \mathrm{O}$ & ${ }^{14} \mathrm{~N}^{18} \mathrm{O}_{3}$ & 68.0011 & $<0.1$ & ${ }^{18} \mathrm{O}^{18} \mathrm{O}^{18} \mathrm{O}$ \\
$\mathrm{M}+7$ & $15 \mathrm{~N} 18 \mathrm{O} 18 \mathrm{O} 18 \mathrm{O}$ & ${ }^{15} \mathrm{~N}^{18} \mathrm{O}_{3}$ & 68.9981 & $<0.1$ & \\
\hline
\end{tabular}




\section{$\delta$-values from analysis with base peak ("with M0")}

Note: all following equations assume negligible effect of clumping/anticlumping on the abundance of singly substituted isotopologues (TODO: example calculation of the potential error introduced).

General equation for $\delta$ value (implicitly x1000 if reported in permil):

$$
{ }^{x / y} \delta=\frac{{ }^{x / y} \mathrm{R}_{\text {sample }}}{x / y \mathrm{R}_{\text {reference }}}-1
$$

$\delta^{15} N$ :

$$
{ }^{15 / 14} \mathrm{R}=\frac{\left[{ }^{15} \mathrm{~N}\right]}{\left[{ }^{14} \mathrm{~N}\right]}=\frac{{ }^{15} \mathrm{~N}^{16} \mathrm{O}_{3}}{{ }^{14} \mathrm{~N}^{16} \mathrm{O}_{3}} \rightarrow \delta^{15} \mathrm{~N}_{\mathrm{sa} / \mathrm{ref}}=\frac{\left(\frac{{ }^{15} \mathrm{~N}^{16} \mathrm{O}_{3}}{{ }^{14} \mathrm{O}_{3}}\right)_{\text {sample }}}{\left(\frac{15 \mathrm{~N}^{16} \mathrm{O}_{3}}{{ }^{14} \mathrm{~N}^{16} \mathrm{O}_{3}}\right)_{\text {reference }}}-1
$$

$\delta^{18} O$ :

$$
{ }^{18 / 16} \mathrm{R}=\frac{\left[{ }^{18} \mathrm{O}\right]}{\left[{ }^{16} \mathrm{O}\right]}=\frac{1}{3} \frac{{ }^{14} \mathrm{~N}^{18} \mathrm{O}^{16} \mathrm{O}_{2}}{{ }^{14} \mathrm{~N}^{16} \mathrm{O}_{3}} \rightarrow \delta^{18} \mathrm{O}_{\mathrm{sa} / \mathrm{ref}}=\frac{\left(\frac{{ }^{14} \mathrm{~N}^{18} \mathrm{O}^{16} \mathrm{O}_{2}}{{ }^{14} \mathrm{~N}^{16} \mathrm{O}_{3}}\right)_{\text {sample }}}{\left(\frac{{ }^{14} \mathrm{~N}^{18} \mathrm{O}^{16} \mathrm{O}_{2}}{{ }^{14} \mathrm{~N}^{16} \mathrm{O}_{3}}\right)_{\text {reference }}}-1
$$

$\delta^{17} O:$

$$
{ }^{17 / 16} \mathrm{R}=\frac{\left[{ }^{17} \mathrm{O}\right]}{\left[{ }^{16} \mathrm{O}\right]}=\frac{1}{3} \frac{{ }^{14} \mathrm{~N}^{17} \mathrm{O}^{16} \mathrm{O}_{2}}{{ }^{14} \mathrm{~N}^{16} \mathrm{O}_{3}} \rightarrow \delta^{17} \mathrm{O}_{\mathrm{sa} / \mathrm{ref}}=\frac{\left(\frac{{ }^{14} \mathrm{~N}^{17} \mathrm{O}^{16} \mathrm{O}_{2}}{{ }^{14} \mathrm{~N}^{16} \mathrm{O}_{3}}\right)_{\text {sample }}}{\left(\frac{{ }^{14} \mathrm{~N}^{17} \mathrm{O}^{16} \mathrm{O}_{2}}{{ }^{14} \mathrm{~N}^{16} \mathrm{O}_{3}}\right)_{\text {reference }}}-1
$$

$\Delta^{17} O:$

$\Delta^{17} \mathrm{O}$ can be calculated from $\delta^{17} \mathrm{O}$ and $\delta^{18} \mathrm{O}$ the usual way with a mass scaling factor $\lambda$ either exact (used everywhere hereafter) or with the linear approximation:

$$
\begin{aligned}
\Delta^{17} \mathrm{O} & =\ln \left(\delta^{17} \mathrm{O}+1\right)-\lambda \cdot \ln \left(\delta^{18} \mathrm{O}+1\right) \\
& \approx \delta^{17} \mathrm{O}-\lambda \cdot \delta^{18} \mathrm{O} \text { for }|\delta|<<1
\end{aligned}
$$

\section{$\delta$-values from analysis without base peak ("without M0")}

$\delta\left({ }^{17} \mathrm{O} /{ }^{18} \mathrm{O}\right)$ :

$$
{ }^{17 / 18} \mathrm{R}=\frac{\left[{ }^{17} \mathrm{O}\right]}{\left[{ }^{18} \mathrm{O}\right]}=\frac{{ }^{14} \mathrm{~N}^{17} \mathrm{O}^{16} \mathrm{O}_{2}}{{ }^{14} \mathrm{~N}^{18} \mathrm{O}^{16} \mathrm{O}_{2}} \rightarrow \delta\left({ }^{17} \mathrm{O} /{ }^{18} \mathrm{O}\right)_{\mathrm{sa} / \mathrm{ref}}=\frac{\left(\frac{{ }^{14} \mathrm{~N}^{17} \mathrm{O}^{16} \mathrm{O}_{2}}{{ }^{14} \mathrm{~N}^{18} \mathrm{O}^{16} \mathrm{O}_{2}}\right)_{\text {sample }}}{\left(\frac{14 \mathrm{~N}^{17} \mathrm{O}^{16} \mathrm{O}_{2}}{{ }^{14} \mathrm{~N}^{18} \mathrm{O}^{16} \mathrm{O}_{2}}\right)_{\text {reference }}}-1
$$

$\delta\left({ }^{18} O /{ }^{15} N\right):$

A less common but useful delta value is the one between $18 \mathrm{O}$ and $15 \mathrm{~N}$ substituted nitrate:

$$
{ }^{18 / 15} \mathrm{R}=\frac{\left[{ }^{18} \mathrm{O}\right] /\left[{ }^{16} \mathrm{O}\right]}{\left[{ }^{15} \mathrm{~N}\right] /\left[{ }^{14} \mathrm{~N}\right]}=\frac{{ }^{14} \mathrm{~N}^{18} \mathrm{O}^{16} \mathrm{O}_{2} /{ }^{14} \mathrm{~N}^{16} \mathrm{O}_{3}}{{ }^{15} \mathrm{~N}^{16} \mathrm{O}_{3} /{ }^{14} \mathrm{~N}^{16} \mathrm{O}_{3}} \rightarrow \delta\left({ }^{18} \mathrm{O} /{ }^{15} \mathrm{~N}\right)_{\mathrm{sa} / \mathrm{ref}}=\frac{\left(\frac{{ }^{14} \mathrm{~N}^{18} \mathrm{O}^{16} \mathrm{O}_{2}}{{ }^{15} \mathrm{~N}^{16} \mathrm{O}_{3}}\right)_{\text {sample }}}{\left(\frac{{ }^{14} \mathrm{~N}^{18} \mathrm{O}^{16} \mathrm{O}_{2}}{{ }^{15} \mathrm{~N}^{16} \mathrm{O}_{3}}\right)_{\text {reference }}}-1
$$


$\delta\left({ }^{17} \mathrm{O} /{ }^{15} \mathrm{~N}\right)$ :

Likewise, the delta value between $17 \mathrm{O}$ and $15 \mathrm{~N}$ substituted nitrate is uncommon but useful:

$$
{ }^{17 / 15} \mathrm{R}=\frac{\left[{ }^{17} \mathrm{O}\right] /\left[{ }^{16} \mathrm{O}\right]}{\left[{ }^{15} \mathrm{~N}\right] /\left[{ }^{14} \mathrm{~N}\right]}=\frac{{ }^{14} \mathrm{~N}^{17} \mathrm{O}^{16} \mathrm{O}_{2} /{ }^{14} \mathrm{~N}^{16} \mathrm{O}_{3}}{{ }^{15} \mathrm{~N}^{16} \mathrm{O}_{3} /{ }^{14} \mathrm{~N}^{16} \mathrm{O}_{3}} \rightarrow \delta\left({ }^{17} \mathrm{O} /{ }^{15} \mathrm{~N}\right)_{\mathrm{sa} / \mathrm{ref}}=\frac{\left(\frac{{ }^{14} \mathrm{~N}^{17} \mathrm{O}^{16} \mathrm{O}_{2}}{{ }^{15} \mathrm{~N}^{16} \mathrm{O}_{3}}\right)_{\text {sample }}}{\left(\frac{{ }^{14} \mathrm{~N}^{17} \mathrm{O}^{16} \mathrm{O}_{2}}{{ }^{15} \mathrm{~N}^{16} \mathrm{O}_{3}}\right)_{\text {reference }}}-1
$$

\section{Possible $\delta$-value calculations}

The following $\delta$ values can be calculated based on a combination of measured $\delta$ values from analyses with M0 and without M0, or from a combination of independently determined $\delta$ values and analysis without M0.

\section{$\delta^{18} O$ revisited:}

Counter-intuitively, if $\delta^{15} \mathrm{~N}$ is already known independently (or from analysis with $\mathrm{M} 0$ ), a $\delta\left({ }^{18} \mathrm{O} /{ }^{15} \mathrm{~N}\right)$ measurement can be used to infer $\delta^{18} \mathrm{O}$ :

$$
\begin{aligned}
\delta^{18} \mathrm{O}_{\mathrm{sa} / \mathrm{ref}} & =\frac{\left(\frac{{ }^{14} \mathrm{~N}^{18} \mathrm{O}^{16} \mathrm{O}_{2}}{{ }^{14} \mathrm{~N}^{16} \mathrm{O}_{3}}\right)_{\text {sample }}}{\left(\frac{{ }^{14} \mathrm{~N}^{18} \mathrm{O}^{16} \mathrm{O}_{2}}{{ }^{14} \mathrm{~N}^{16} \mathrm{O}_{3}}\right)_{\text {reference }}}-1=\frac{\left(\frac{{ }^{14} \mathrm{~N}^{18} \mathrm{O}^{16} \mathrm{O}_{2}}{{ }^{15} \mathrm{~N}^{16} \mathrm{O}_{3}} \frac{{ }^{15} \mathrm{~N}^{16} \mathrm{O}_{3}}{{ }^{14} \mathrm{~N}^{16} \mathrm{O}_{3}}\right)_{\text {sample }}}{\left(\frac{{ }^{14} \mathrm{~N}^{18} \mathrm{O}^{16} \mathrm{O}_{2}}{{ }^{15} \mathrm{~N}^{16} \mathrm{O}_{3}} \frac{\mathrm{N}^{16} \mathrm{O}_{3}}{14 \mathrm{~N}^{16} \mathrm{O}_{3}}\right)_{\text {reference }}}-1 \\
= & \left(\delta\left({ }^{18} \mathrm{O} /{ }^{15} \mathrm{~N}\right)_{\mathrm{sa} / \mathrm{ref}}+1\right)\left(\delta^{15} \mathrm{~N}_{\mathrm{sa} / \mathrm{ref}}+1\right)-1 \\
& =\delta\left({ }^{18} \mathrm{O} /{ }^{15} \mathrm{~N}\right)_{\mathrm{sa} / \mathrm{ref}}+\delta^{15} \mathrm{~N}_{\mathrm{sa} / \mathrm{ref}}+\delta\left({ }^{18} \mathrm{O} /{ }^{15} \mathrm{~N}\right)_{\mathrm{sa} / \mathrm{ref}} \cdot \delta^{15} \mathrm{~N}_{\mathrm{sa} / \mathrm{ref}}
\end{aligned}
$$

$\delta^{15} N$ revisited:

Inversely, $\delta^{15} \mathrm{~N}$ can be inferred if $\delta^{18} \mathrm{O}$ is already known:

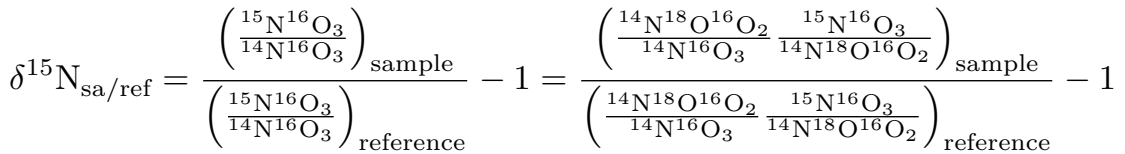

$$
\begin{aligned}
& =\frac{\delta^{18} \mathrm{O}_{\mathrm{sa} / \mathrm{ref}}+1}{\delta\left({ }^{18} \mathrm{O} /{ }^{15} \mathrm{~N}\right)_{\mathrm{sa} / \mathrm{ref}}+1}-1 \\
& =\frac{\delta^{18} \mathrm{O}_{\mathrm{sa} / \mathrm{ref}}-\delta\left({ }^{18} \mathrm{O} /{ }^{15} \mathrm{~N}\right)_{\mathrm{sa} / \mathrm{ref}}}{\delta\left({ }^{18} \mathrm{O} /{ }^{15} \mathrm{~N}\right)_{\mathrm{sa} / \mathrm{ref}}+1}
\end{aligned}
$$

\section{$\delta^{17} O$ revisited:}

Likewise, $\delta^{17} \mathrm{O}$ can be inferred from analysis without M0 if $\delta^{15} \mathrm{~N}$ OR $\delta^{18} \mathrm{O}$ are already known independently (or from analysis with M0). With $\delta^{15} \mathrm{~N}$ :

$$
\begin{aligned}
& \delta^{17} \mathrm{O}_{\mathrm{sa} / \mathrm{ref}}=\frac{\left(\frac{{ }^{14} \mathrm{~N}^{17} \mathrm{O}^{16} \mathrm{O}_{2}}{{ }^{14} \mathrm{~N}^{16} \mathrm{O}_{3}}\right)_{\text {sample }}}{\left(\frac{{ }^{14} \mathrm{~N}^{17} \mathrm{O}^{16} \mathrm{O}_{2}}{{ }^{14} \mathrm{~N}^{16} \mathrm{O}_{3}}\right)_{\text {reference }}}-1=\frac{\left(\frac{{ }^{14} \mathrm{~N}^{17} \mathrm{O}^{16} \mathrm{O}_{2}}{{ }^{15} \mathrm{~N}^{16} \mathrm{O}_{3}} \frac{{ }^{15} \mathrm{~N}^{16} \mathrm{O}_{3}}{{ }^{14} \mathrm{~N}^{16} \mathrm{O}_{3}}\right)_{\text {sample }}}{\left(\frac{{ }^{14} \mathrm{~N}^{17} \mathrm{O}^{16} \mathrm{O}_{2}}{{ }^{15} \mathrm{~N}^{16} \mathrm{O}_{3}} \frac{15 \mathrm{~N}^{16} \mathrm{O}_{3}}{14 \mathrm{~N}^{16} \mathrm{O}_{3}}\right)_{\text {reference }}}-1 \\
& =\left(\delta\left({ }^{17} \mathrm{O} /{ }^{15} \mathrm{~N}\right)_{\mathrm{sa} / \mathrm{ref}}+1\right)\left(\delta^{15} \mathrm{~N}_{\mathrm{sa} / \mathrm{ref}}+1\right)-1 \\
& =\delta\left({ }^{17} \mathrm{O} /{ }^{15} \mathrm{~N}\right)_{\mathrm{sa} / \mathrm{ref}}+\delta^{15} \mathrm{~N}_{\mathrm{sa} / \mathrm{ref}}+\delta\left({ }^{17} \mathrm{O} /{ }^{15} \mathrm{~N}\right)_{\mathrm{sa} / \mathrm{ref}} \cdot \delta^{15} \mathrm{~N}_{\mathrm{sa} / \mathrm{ref}}
\end{aligned}
$$


or with $\delta^{18} \mathrm{O}$ :

$$
\begin{aligned}
\delta^{17} \mathrm{O}_{\mathrm{sa} / \mathrm{ref}} & =\frac{\left(\frac{{ }^{14} \mathrm{~N}^{17} \mathrm{O}^{16} \mathrm{O}_{2}}{{ }^{14} \mathrm{~N}^{16} \mathrm{O}_{3}}\right)_{\text {sample }}}{\left(\frac{{ }^{14} \mathrm{~N}^{17} \mathrm{O}^{16} \mathrm{O}_{2}}{{ }^{14} \mathrm{~N}^{16} \mathrm{O}_{3}}\right)_{\text {reference }}}-1=\frac{\left(\frac{{ }^{14} \mathrm{~N}^{18} \mathrm{O}^{16} \mathrm{O}_{2}}{{ }^{14} \mathrm{~N}^{16} \mathrm{O}_{3}} \frac{{ }^{14} \mathrm{~N}^{17} \mathrm{O}^{16} \mathrm{O}_{2}}{{ }^{18} \mathrm{O}^{16} \mathrm{O}_{2}}\right)_{\text {sample }}}{\left(\frac{{ }^{14} \mathrm{~N}^{18} \mathrm{O}^{16} \mathrm{O}_{2}}{{ }^{14} \mathrm{~N}^{16} \mathrm{O}_{3}} \frac{14 \mathrm{~N}^{17} \mathrm{O}^{16} \mathrm{O}_{2}}{14 \mathrm{~N}^{18} \mathrm{O}^{16} \mathrm{O}_{2}}\right)_{\text {reference }}}-1 \\
= & \left(\delta\left({ }^{17} \mathrm{O} /{ }^{18} \mathrm{O}\right)_{\mathrm{sa} / \mathrm{ref}}+1\right)\left(\delta^{18} \mathrm{O}_{\mathrm{sa} / \mathrm{ref}}+1\right)-1 \\
= & \delta\left({ }^{17} \mathrm{O} /{ }^{18} \mathrm{O}\right)_{\mathrm{sa} / \mathrm{ref}}+\delta^{18} \mathrm{O}_{\mathrm{sa} / \mathrm{ref}}+\delta\left({ }^{17} \mathrm{O} /{ }^{18} \mathrm{O}\right)_{\mathrm{sa} / \mathrm{ref}} \cdot \delta^{18} \mathrm{O}_{\mathrm{sa} / \mathrm{ref}}
\end{aligned}
$$

\section{$\Delta^{17} O$ revisited:}

Based on the above $\delta^{17} \mathrm{O}$ calculations, $\Delta^{17} \mathrm{O}$ can likewise be calculated with $\delta^{18} \mathrm{O}$ as:

$$
\begin{aligned}
\Delta^{17} \mathrm{O} & =\ln \left(\delta^{17} \mathrm{O}+1\right)-\lambda \cdot \ln \left(\delta^{18} \mathrm{O}+1\right) \\
& =\ln \left(\left(\delta^{18} \mathrm{O}+1\right)\left(\delta\left({ }^{17} \mathrm{O} /{ }^{18} \mathrm{O}\right)+1\right)\right)-\lambda \cdot \ln \left(\delta^{18} \mathrm{O}+1\right) \\
& =\ln \left(\delta^{18} \mathrm{O}+1\right)+\ln \left(\delta\left({ }^{17} \mathrm{O} /{ }^{18} \mathrm{O}\right)+1\right)-\lambda \cdot \ln \left(\delta^{18} \mathrm{O}+1\right) \\
& =\ln \left(\delta\left({ }^{17} \mathrm{O} /{ }^{18} \mathrm{O}\right)+1\right)+(1-\lambda) \cdot \ln \left(\delta^{18} \mathrm{O}+1\right)
\end{aligned}
$$

or even with $\delta^{15} \mathrm{~N}$ :

$$
\begin{aligned}
\Delta^{17} \mathrm{O} & =\ln \left(\delta^{17} \mathrm{O}+1\right)-\lambda \cdot \ln \left(\delta^{18} \mathrm{O}+1\right) \\
& =\ln \left(\left(\delta^{15} \mathrm{~N}+1\right)\left(\delta\left({ }^{17} \mathrm{O} /{ }^{15} \mathrm{~N}\right)+1\right)\right)-\lambda \cdot \ln \left(\left(\delta^{15} \mathrm{~N}+1\right)\left(\delta\left({ }^{18} \mathrm{O} /{ }^{15} \mathrm{~N}\right)+1\right)\right) \\
& =\ln \left(\delta^{15} \mathrm{~N}+1\right)+\ln \left(\delta\left({ }^{17} \mathrm{O} /{ }^{15} \mathrm{~N}\right)+1\right)-\lambda \cdot \ln \left(\delta^{15} \mathrm{~N}+1\right)-\lambda \cdot \ln \left(\delta\left({ }^{18} \mathrm{O} /{ }^{15} \mathrm{~N}\right)+1\right) \\
& =(1-\lambda) \cdot \ln \left(\delta^{15} \mathrm{~N}+1\right)+\ln \left(\delta\left({ }^{17} \mathrm{O} /{ }^{15} \mathrm{~N}\right)+1\right)-\lambda \cdot \ln \left(\delta\left({ }^{18} \mathrm{O} /{ }^{15} \mathrm{~N}\right)+1\right)
\end{aligned}
$$




\section{Clumped isotopes}

General equation for clumped $\Delta$ values (usually x1000 for permil, not the same as mass-independent $\Delta !$ ) for an isotope exchange reaction with two heavy isotopes $(H)$ :

$$
\begin{gathered}
H L+L H \leftrightarrow H H+L L \\
Q=\frac{[H H][L L]}{[H L][L H]} \\
\Delta H H=\ln (Q) \approx \frac{m_{H H} / m_{L L} \mathrm{R}_{\text {measured }}}{m_{H H} / m_{L L} \mathrm{R}_{\text {stochastic }}}-1
\end{gathered}
$$

where $m_{H H}$ and $m_{L L}$ are the masses of the doubly substituted and unsubstituted isotopologues, respectively.

$\Delta^{15} N^{18} O$ :

Reaction and $\Delta$ :

$$
\begin{aligned}
{ }^{15} \mathrm{~N}^{16} \mathrm{O}_{3}^{-}+{ }^{14} \mathrm{~N}^{18} \mathrm{O}^{16} \mathrm{O}_{2}^{-} & \leftrightarrow{ }^{15} \mathrm{~N}^{18} \mathrm{O}^{16} \mathrm{O}_{2}^{-}+{ }^{14} \mathrm{~N}^{16} \mathrm{O}_{3}^{-} \\
\rightarrow \Delta^{15} \mathrm{~N}^{18} \mathrm{O} & =\frac{65 / 62}{\mathrm{R}_{\text {measured }}}-1
\end{aligned}
$$

\section{Stochastic R:}

$$
\begin{aligned}
{ }^{65 / 62} \mathrm{R}_{\text {stochastic }} & =\frac{{ }^{15} \mathrm{~N}^{18} \mathrm{O}^{16} \mathrm{O}^{16} \mathrm{O}^{-}+{ }^{15} \mathrm{~N}^{16} \mathrm{O}^{18} \mathrm{O}^{16} \mathrm{O}^{-}+{ }^{15} \mathrm{~N}^{16} \mathrm{O}^{16} \mathrm{O}^{18} \mathrm{O}^{-}}{{ }^{14} \mathrm{~N}^{16} \mathrm{O}^{16} \mathrm{O}^{16} \mathrm{O}^{-}}=\frac{3 \cdot\left[{ }^{15} \mathrm{~N}\right] \cdot\left[{ }^{18} \mathrm{O}\right] \cdot\left[{ }^{16} \mathrm{O}\right]^{2}}{\left[{ }^{14} \mathrm{~N}\right] \cdot\left[{ }^{16} \mathrm{O}\right]^{3}} \\
& =3 \cdot{ }^{15 / 14} \mathrm{R} \cdot{ }^{18 / 16} \mathrm{R} \\
\rightarrow \Delta^{15} \mathrm{~N}^{18} \mathrm{O} & =\frac{{ }^{65 / 62} \mathrm{R}}{3 \cdot{ }^{15 / 14} \mathrm{R} \cdot{ }^{18 / 16} \mathrm{R}}-1
\end{aligned}
$$

\section{Measured R (multiple ways to calculate the same):}

using 65/63 ratio :

$$
\begin{gathered}
{ }^{65 / 62} \mathrm{R}_{\text {measured }}=\frac{{ }^{15} \mathrm{~N}^{18} \mathrm{O}^{16} \mathrm{O}_{2}}{{ }^{14} \mathrm{~N}^{16} \mathrm{O}_{3}}=\frac{{ }^{15} \mathrm{~N}^{18} \mathrm{O}^{16} \mathrm{O}_{2}}{{ }^{15} \mathrm{~N}^{16} \mathrm{O}_{3}} \cdot \frac{{ }^{15} \mathrm{~N}^{16} \mathrm{O}_{3}}{{ }^{14} \mathrm{~N}^{16} \mathrm{O}_{3}}=\frac{{ }^{15} \mathrm{~N}^{18} \mathrm{O}^{16} \mathrm{O}_{2}}{{ }^{15} \mathrm{~N}^{16} \mathrm{O}_{3}} \cdot{ }^{15 / 14} \mathrm{R} \\
\rightarrow \Delta^{15} \mathrm{~N}^{18} \mathrm{O}=\frac{\frac{{ }^{15} \mathrm{~N}^{18} \mathrm{O}^{16} \mathrm{O}_{2}}{{ }^{15} \mathrm{~N}^{16} \mathrm{O}_{3}} \cdot{ }^{15 / 14} \mathrm{R}}{3 \cdot{ }^{15 / 14} \mathrm{R} \cdot{ }^{18 / 16} \mathrm{R}}-1=\frac{{ }^{15} \mathrm{~N}^{18} \mathrm{O}^{16} \mathrm{O}_{2}}{{ }^{15} \mathrm{~N}^{16} \mathrm{O}_{3}} \cdot \frac{1}{3 \cdot{ }^{18 / 16} \mathrm{R}}-1
\end{gathered}
$$

using 65/64 ratio :

$$
\begin{aligned}
& { }^{65 / 62} \mathrm{R}_{\text {measured }}=\frac{{ }^{15} \mathrm{~N}^{18} \mathrm{O}^{16} \mathrm{O}_{2}}{{ }^{14} \mathrm{~N}^{16} \mathrm{O}_{3}}=\frac{{ }^{15} \mathrm{~N}^{18} \mathrm{O}^{16} \mathrm{O}_{2}}{{ }^{14} \mathrm{~N}^{18} \mathrm{O}^{16} \mathrm{O}_{2}} \cdot \frac{{ }^{14} \mathrm{~N}^{18} \mathrm{O}^{16} \mathrm{O}_{2}}{{ }^{14} \mathrm{~N}^{16} \mathrm{O}_{3}}=\frac{{ }^{15} \mathrm{~N}^{18} \mathrm{O}^{16} \mathrm{O}_{2}}{{ }^{14} \mathrm{~N}^{18} \mathrm{O}^{16} \mathrm{O}_{2}} \cdot{ }^{18 / 16} \mathrm{R}
\end{aligned}
$$

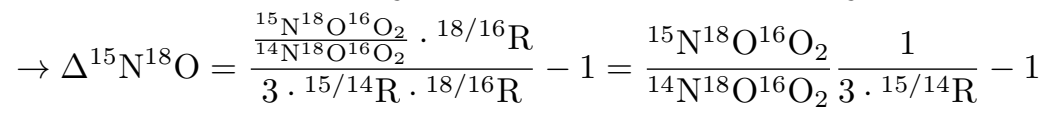

$\epsilon^{15} N^{18} O$ :

We can determine absolute $\Delta^{15} \mathrm{~N}^{18} \mathrm{O}$ from various combinations of $\mathrm{M} 0$ and $\mathrm{M}+1$ analysis as outlined above. Alternatively, we can compare two materials (here called "sample" and "reference") to measure relative clumping/anticlumping which may be more practical for analytical comparison and standardization. This is akin to a fractionation factor $\epsilon$ and could be calculated in different ways from the various peaks measured in M+1 mode, $\delta^{15} \mathrm{~N}$ 
and $\delta^{18} \mathrm{O}$ (either measured in M0 mode or independently determined) as long as each value is measured against the same reference material

$$
\epsilon^{15} \mathrm{~N}^{18} \mathrm{O}_{\mathrm{sa} / \mathrm{ref}}=\frac{\left(\frac{65 / 62 \mathrm{R}_{\text {measured }}}{65 / 62 \mathrm{R}_{\text {stochastic }}}\right)_{\text {sample }}}{\left(\frac{65 / 62}{6_{\text {measured }}}\right)_{\text {reference }}}-1=\frac{\left(\Delta^{15} \mathrm{~N}^{18} \mathrm{O}+1\right)_{\text {sample }}}{\left(\Delta^{15} \mathrm{~N}^{18} \mathrm{O}+1\right)_{\text {referenche }}}-1
$$

using $\delta\left({ }^{15} \mathrm{~N}^{18} \mathrm{O} /{ }^{15} \mathrm{~N}\right)$ :

$$
\begin{aligned}
\epsilon^{15} \mathrm{~N}^{18} \mathrm{O}_{\mathrm{sa} / \mathrm{ref}} & =\frac{\delta\left({ }^{15} \mathrm{~N}^{18} \mathrm{O} /{ }^{15} \mathrm{~N}\right)_{\mathrm{sa} / \mathrm{ref}}+1}{\delta^{18} \mathrm{O}_{\mathrm{sa} / \mathrm{ref}}+1}-1 \\
& {\left[\text { with } \delta\left({ }^{15} \mathrm{~N}^{18} \mathrm{O} /{ }^{15} \mathrm{~N}\right)_{\mathrm{sa} / \mathrm{ref}}=\frac{\left(\frac{{ }^{15} \mathrm{~N}^{18} \mathrm{O}^{16} \mathrm{O}_{2}}{{ }^{15} \mathrm{~N}^{16} \mathrm{O}_{3}}\right)_{\text {sample }}}{\left(\frac{{ }^{15} \mathrm{~N}^{18} \mathrm{O}^{16} \mathrm{O}_{2}}{{ }^{15} \mathrm{~N}^{16} \mathrm{O}_{3}}\right)_{\text {reference }}}-1\right] }
\end{aligned}
$$

using $\delta\left({ }^{15} \mathrm{~N}^{18} \mathrm{O} /{ }^{18} \mathrm{O}\right)$ :

$$
\begin{aligned}
\epsilon^{15} \mathrm{~N}^{18} \mathrm{O}_{\mathrm{sa} / \mathrm{ref}} & =\frac{\delta\left({ }^{15} \mathrm{~N}^{18} \mathrm{O} /{ }^{18} \mathrm{O}\right)_{\mathrm{sa} / \mathrm{ref}}+1}{\delta^{15} \mathrm{~N}_{\mathrm{sa} / \mathrm{ref}}+1}-1 \\
& {\left[\text { with } \delta\left({ }^{15} \mathrm{~N}^{18} \mathrm{O} /{ }^{18} \mathrm{O}\right)_{\mathrm{sa} / \mathrm{ref}}=\frac{\left(\frac{\left(15 \mathrm{~N}^{18} \mathrm{O}^{16} \mathrm{O}_{2}\right.}{{ }^{14} \mathrm{~N}^{18} \mathrm{O}^{16} \mathrm{O}_{2}}\right)_{\text {sample }}}{\left(\frac{15 \mathrm{~N}^{18} \mathrm{O}^{16} \mathrm{O}_{2}}{{ }^{14} \mathrm{~N}^{18} \mathrm{O}^{16} \mathrm{O}_{2}}\right)_{\text {reference }}}-1\right] }
\end{aligned}
$$

\section{$\delta^{18} O$ and $\delta^{15} N$ revisited:}

The above equations also highlight the following useful relationships for $\delta^{18} O$

$$
\begin{aligned}
\frac{\left(\Delta^{15} \mathrm{~N}^{18} \mathrm{O}+1\right)_{\text {sample }}}{\left(\Delta^{15} \mathrm{~N}^{18} \mathrm{O}+1\right)_{\text {reference }}} & =\frac{\delta\left({ }^{15} \mathrm{~N}^{18} \mathrm{O} /{ }^{15} \mathrm{~N}\right)_{\mathrm{sa} / \mathrm{ref}}+1}{\delta^{18} \mathrm{O}_{\mathrm{sa} / \mathrm{ref}}+1} \\
\rightarrow \delta^{18} \mathrm{O}_{\mathrm{sa} / \mathrm{ref}} & =\left(\delta\left({ }^{15} \mathrm{~N}^{18} \mathrm{O} /{ }^{15} \mathrm{~N}\right)_{\mathrm{sa} / \mathrm{ref}}+1\right) \cdot \frac{\left(\Delta^{15} \mathrm{~N}^{18} \mathrm{O}+1\right)_{\text {ref }}}{\left(\Delta^{15} \mathrm{~N}^{18} \mathrm{O}+1\right)_{\text {sample }}}-1 \\
\rightarrow \delta^{18} \mathrm{O}_{\mathrm{sa} / \mathrm{ref}} & =\delta\left({ }^{15} \mathrm{~N}^{18} \mathrm{O} /{ }^{15} \mathrm{~N}\right)_{\mathrm{sa} / \mathrm{ref}} \mid \text { if } \Delta^{15} \mathrm{~N}^{18} \mathrm{O}_{\mathrm{ref}}=\Delta^{15} \mathrm{~N}^{18} \mathrm{O}_{\text {sample }}
\end{aligned}
$$

and $\delta^{15} N$ :

$$
\begin{aligned}
\frac{\left(\Delta^{15} \mathrm{~N}^{18} \mathrm{O}+1\right)_{\text {sample }}}{\left(\Delta^{15} \mathrm{~N}^{18} \mathrm{O}+1\right)_{\text {reference }}} & =\frac{\delta\left({ }^{15} \mathrm{~N}^{18} \mathrm{O} /{ }^{18} \mathrm{O}\right)_{\mathrm{sa} / \mathrm{ref}}+1}{\delta^{15} \mathrm{~N}_{\mathrm{sa} / \mathrm{ref}}+1} \\
\rightarrow \delta^{15} \mathrm{~N}_{\mathrm{sa} / \mathrm{ref}} & =\left(\delta\left({ }^{15} \mathrm{~N}^{18} \mathrm{O} /{ }^{18} \mathrm{O}\right)_{\mathrm{sa} / \text { ref }}+1\right) \cdot \frac{\left(\Delta^{15} \mathrm{~N}^{18} \mathrm{O}+1\right)_{\text {ref }}}{\left(\Delta^{15} \mathrm{~N}^{18} \mathrm{O}+1\right)_{\text {sample }}}-1 \\
\rightarrow \delta^{15} \mathrm{~N}_{\mathrm{sa} / \mathrm{ref}} & =\delta\left({ }^{15} \mathrm{~N}^{18} \mathrm{O} /{ }^{18} \mathrm{O}\right)_{\mathrm{sa} / \mathrm{ref}} \mid \text { if } \Delta^{15} \mathrm{~N}^{18} \mathrm{O}_{\text {ref }}=\Delta^{15} \mathrm{~N}^{18} \mathrm{O}_{\text {sample }}
\end{aligned}
$$

$\Delta^{18} \mathrm{O}^{18} \mathrm{O}:$

Identical equations can be derived for the ${ }^{18} \mathrm{O}^{18} \mathrm{O}$ clump (simpler because of the single element):

\section{Reaction and $\Delta$ :}

$$
\begin{aligned}
{ }^{14} \mathrm{~N}^{18} \mathrm{O}^{16} \mathrm{O}_{2}^{-}+{ }^{14} \mathrm{~N}^{18} \mathrm{O}^{16} \mathrm{O}_{2}^{-} & \leftrightarrow{ }^{14} \mathrm{~N}^{18} \mathrm{O}^{216} \mathrm{O}^{-}+{ }^{14} \mathrm{~N}^{16} \mathrm{O}_{3}^{-} \\
\rightarrow \Delta^{18} \mathrm{O}^{18} \mathrm{O} & =\frac{{ }^{66} \mathrm{R}_{\text {measured }}}{{ }^{66 / 62} \mathrm{R}_{\text {stochastic }}}-1
\end{aligned}
$$




\section{Stochastic R:}

$$
\begin{aligned}
{ }^{66 / 62} \mathrm{R}_{\text {stochastic }} & \left.=\frac{{ }^{14} \mathrm{~N}^{18} \mathrm{O}^{18} \mathrm{O}^{16} \mathrm{O}^{-}+{ }^{14} \mathrm{~N}^{16} \mathrm{O}^{18} \mathrm{O}^{18} \mathrm{O}^{-}+{ }^{14} \mathrm{~N}^{18} \mathrm{O}^{16} \mathrm{O}^{18} \mathrm{O}^{-}}{{ }^{14} \mathrm{~N}^{16} \mathrm{O}^{16} \mathrm{O}^{16} \mathrm{O}^{-}}=\frac{3 \cdot\left[{ }^{14} \mathrm{~N}\right] \cdot\left[{ }^{18} \mathrm{O}\right]{ }^{2} \cdot\left[{ }^{16} \mathrm{O}\right]}{\left[{ }^{14} \mathrm{~N}\right] \cdot\left[{ }^{16} \mathrm{O}\right]}\right]^{3} \\
& =3 \cdot\left({ }^{18 / 16} \mathrm{R}\right)^{2} \\
\rightarrow \Delta^{18} \mathrm{O}^{18} \mathrm{O} & =\frac{{ }^{66 / 62} \mathrm{R}}{3 \cdot\left({ }^{18 / 16} \mathrm{R}\right)^{2}}-1
\end{aligned}
$$

Measured R (multiple ways to calculate the same):

$$
\begin{aligned}
& { }^{66 / 62} \mathrm{R}_{\text {measured }}=\frac{{ }^{14} \mathrm{~N}^{18} \mathrm{O}_{2}{ }^{16} \mathrm{O}}{{ }^{14} \mathrm{~N}^{16} \mathrm{O}_{3}}=\frac{{ }^{14} \mathrm{~N}^{18} \mathrm{O}_{2}{ }^{16} \mathrm{O}}{{ }^{14} \mathrm{~N}^{18} \mathrm{O}^{16} \mathrm{O}_{2}} \cdot \frac{{ }^{14} \mathrm{~N}^{18} \mathrm{O}^{16} \mathrm{O}_{2}}{{ }^{14} \mathrm{~N}^{16} \mathrm{O}_{3}}=\frac{{ }^{14} \mathrm{~N}^{18} \mathrm{O}_{2}{ }^{16} \mathrm{O}}{{ }^{14} \mathrm{~N}^{18} \mathrm{O}^{16} \mathrm{O}_{2}} \cdot 3 \cdot{ }^{18 / 16} \mathrm{R} \\
& \rightarrow \Delta^{18} \mathrm{O}^{18} \mathrm{O}=\frac{\frac{{ }^{14} \mathrm{~N}^{18} \mathrm{O}_{2}{ }^{16} \mathrm{O}}{{ }^{14} \mathrm{~N}^{18} \mathrm{O}^{16} \mathrm{O}_{2}} \cdot 3 \cdot{ }^{18 / 16} \mathrm{R}}{3 \cdot\left({ }^{18 / 16} \mathrm{R}\right)^{2}}-1=\frac{{ }^{14} \mathrm{~N}^{18} \mathrm{O}_{2}{ }^{16} \mathrm{O}}{{ }^{14} \mathrm{~N}^{18} \mathrm{O}^{16} \mathrm{O}_{2}} \cdot \frac{1}{{ }^{18 / 16} \mathrm{R}}-1
\end{aligned}
$$

$\epsilon^{18} O^{18} O$

$$
\begin{aligned}
\left.\epsilon^{18} \mathrm{O}^{18} \mathrm{O}=\frac{\left(\frac{66 / 62}{66 / 62} \mathrm{R}_{\text {stoasured }}\right.}{\left(\frac{66 / 62}{{ }^{6} \mathrm{R}_{\text {measured }}}\right)_{\text {sample }}}\right)_{\text {reference }}-1= & \frac{\left(\Delta^{18} \mathrm{O}^{18} \mathrm{O}+1\right)_{\text {sample }}}{\left(\Delta^{18} \mathrm{O}^{18} \mathrm{O}+1\right)_{\text {reference }}}-1=\frac{\delta\left({ }^{18} \mathrm{O}^{18} \mathrm{O} /{ }^{18} \mathrm{O}\right)_{\text {sa } / \text { ref }}+1}{\delta^{18} \mathrm{O}_{\mathrm{sa} / \mathrm{ref}}+1}-1 \\
& {\left[\text { with } \delta\left({ }^{18} \mathrm{O}^{18} \mathrm{O} /{ }^{18} \mathrm{O}\right)_{\mathrm{sa} / \mathrm{ref}}=\frac{\left(\frac{{ }^{14} \mathrm{~N}^{18} \mathrm{O}_{2}{ }^{16} \mathrm{O}}{{ }^{14} \mathrm{~N}^{18} \mathrm{O}^{16} \mathrm{O}_{2}}\right)_{\text {sample }}}{\left(\frac{14 \mathrm{~N}^{18} \mathrm{O}_{2}{ }^{16} \mathrm{O}}{{ }^{14} \mathrm{~N}^{18} \mathrm{O}^{16} \mathrm{O}_{2}}\right)_{\text {reference }}}-1\right] }
\end{aligned}
$$

\section{$\delta^{18} O$ revisited:}

The above equation likewise highlights the following useful relationships for $\delta^{18} O$ :

$$
\begin{aligned}
\frac{\left(\Delta^{18} \mathrm{O}^{18} \mathrm{O}+1\right)_{\text {sample }}}{\left(\Delta^{18} \mathrm{O}^{18} \mathrm{O}+1\right)_{\text {reference }}} & =\frac{\delta\left({ }^{18} \mathrm{O}^{18} \mathrm{O} /{ }^{18} \mathrm{O}\right)_{\mathrm{sa} / \mathrm{ref}}+1}{\delta^{18} \mathrm{O}_{\mathrm{sa} / \mathrm{ref}}+1} \\
\rightarrow \delta^{18} \mathrm{O}_{\mathrm{sa} / \mathrm{ref}} & =\left(\delta\left({ }^{18} \mathrm{O}^{18} \mathrm{O} /{ }^{18} \mathrm{O}\right)_{\mathrm{sa} / \mathrm{ref}}+1\right) \cdot \frac{\left(\Delta^{18} \mathrm{O}^{18} \mathrm{O}+1\right)_{\text {ref }}}{\left(\Delta^{18} \mathrm{O}^{18} \mathrm{O}+1\right)_{\text {sample }}}-1 \\
\rightarrow \delta^{18} \mathrm{O}_{\mathrm{sa} / \mathrm{ref}} & =\delta\left({ }^{18} \mathrm{O}^{18} \mathrm{O} /{ }^{18} \mathrm{O}\right)_{\mathrm{sa} / \mathrm{ref}} \mid \text { if } \Delta^{18} \mathrm{O}^{18} \mathrm{O}_{\mathrm{ref}}=\Delta^{18} \mathrm{O}^{18} \mathrm{O}_{\text {sample }}
\end{aligned}
$$


Summary for $\delta^{15} N, \delta^{18} O, \delta^{17} O, \Delta^{17} O$

All $\delta$ value calculations that involve more than one $\delta$ value require measurements for all $\delta$ s to be between the same sample and reference.

$\delta^{15} N$ :

$$
\begin{aligned}
& \delta^{15} \mathrm{~N}_{\mathrm{sa} / \mathrm{ref}}=\frac{\left(\frac{15 \mathrm{~N}^{16} \mathrm{O}_{3}}{{ }^{14} \mathrm{~N}^{16} \mathrm{O}_{3}}\right)_{\text {sample }}}{\left(\frac{15 \mathrm{~N}^{16} \mathrm{O}_{3}}{{ }^{14} \mathrm{~N}^{16} \mathrm{O}_{3}}\right)_{\text {reference }}}-1 \\
& \delta^{15} \mathrm{~N}_{\mathrm{sa} / \mathrm{ref}}=\frac{\delta^{18} \mathrm{O}_{\mathrm{sa} / \mathrm{ref}}-\delta\left({ }^{18} \mathrm{O} /{ }^{15} \mathrm{~N}\right)_{\mathrm{sa} / \mathrm{ref}}}{\delta\left({ }^{18} \mathrm{O} /{ }^{15} \mathrm{~N}\right)_{\mathrm{sa} / \mathrm{ref}}+1} \\
& \text { with } \delta\left({ }^{18} \mathrm{O} /{ }^{15} \mathrm{~N}\right)_{\mathrm{sa} / \mathrm{ref}}=\frac{\left(\frac{{ }^{14} \mathrm{~N}^{18} \mathrm{O}^{16} \mathrm{O}_{2}}{{ }^{15} \mathrm{~N}^{16} \mathrm{O}_{3}}\right)_{\text {sample }}}{\left(\frac{{ }^{14} \mathrm{~N}^{18} \mathrm{O}^{16} \mathrm{O}_{2}}{{ }^{15} \mathrm{~N}^{16} \mathrm{O}_{3}}\right)_{\text {reference }}}-1 \\
& \delta^{15} \mathrm{~N}_{\mathrm{sa} / \mathrm{ref}}=\delta\left({ }^{15} \mathrm{~N}^{18} \mathrm{O} /{ }^{18} \mathrm{O}\right)_{\mathrm{sa} / \mathrm{ref}}=\frac{\left(\frac{15 \mathrm{~N}^{18} \mathrm{O}^{16} \mathrm{O}_{2}}{{ }^{14} \mathrm{~N}^{18} \mathrm{O}^{16} \mathrm{O}_{2}}\right)_{\text {sample }}}{\left(\frac{15 \mathrm{~N}^{18} \mathrm{O}^{16} \mathrm{O}_{2}}{{ }^{14} \mathrm{~N}^{18} \mathrm{O}^{16} \mathrm{O}_{2}}\right)_{\text {reference }}}-1 \\
& \text { if } \Delta^{15} \mathrm{~N}^{18} \mathrm{O}_{\text {ref }}=\Delta^{15} \mathrm{~N}^{18} \mathrm{O}_{\text {sample }}
\end{aligned}
$$

$\delta^{18} O:$

$$
\begin{aligned}
& \delta^{18} \mathrm{O}_{\mathrm{sa} / \mathrm{ref}}=\frac{\left(\frac{{ }^{14} \mathrm{~N}^{18} \mathrm{O}^{16} \mathrm{O}_{2}}{{ }^{14} \mathrm{~N}^{16} \mathrm{O}_{3}}\right)_{\text {sample }}}{\left(\frac{{ }^{14} \mathrm{~N}^{18} \mathrm{O}^{16} \mathrm{O}_{2}}{{ }^{14} \mathrm{~N}^{16} \mathrm{O}_{3}}\right)_{\text {reference }}}-1 \\
& \delta^{18} \mathrm{O}_{\mathrm{sa} / \mathrm{ref}}=\delta\left({ }^{18} \mathrm{O} /{ }^{15} \mathrm{~N}\right)_{\mathrm{sa} / \mathrm{ref}}+\delta^{15} \mathrm{~N}_{\mathrm{sa} / \mathrm{ref}}+\delta\left({ }^{18} \mathrm{O} /{ }^{15} \mathrm{~N}\right)_{\mathrm{sa} / \mathrm{ref}} \cdot \delta^{15} \mathrm{~N}_{\mathrm{sa} / \mathrm{ref}} \\
& \text { with } \delta\left({ }^{18} \mathrm{O} /{ }^{15} \mathrm{~N}\right)_{\mathrm{sa} / \mathrm{ref}}=\frac{\left(\frac{14 \mathrm{~N}^{18} \mathrm{O}^{16} \mathrm{O}_{2}}{{ }^{15} \mathrm{~N}^{16} \mathrm{O}_{3}}\right)_{\text {sample }}}{\left(\frac{14 \mathrm{~N}^{18} \mathrm{O}^{16} \mathrm{O}_{2}}{{ }^{15} \mathrm{~N}^{16} \mathrm{O}_{3}}\right)_{\text {reference }}}-1 \\
& \delta^{18} \mathrm{O}_{\mathrm{sa} / \mathrm{ref}}=\delta\left({ }^{15} \mathrm{~N}^{18} \mathrm{O} /{ }^{15} \mathrm{~N}\right)_{\mathrm{sa} / \mathrm{ref}}=\frac{\left(\frac{15 \mathrm{~N}^{18} \mathrm{O}^{16} \mathrm{O}_{2}}{{ }^{15} \mathrm{~N}^{16} \mathrm{O}_{3}}\right)_{\text {sample }}}{\left(\frac{15 \mathrm{~N}^{18} \mathrm{O}^{16} \mathrm{O}_{2}}{{ }^{15} \mathrm{~N}^{16} \mathrm{O}_{3}}\right)_{\text {reference }}}-1 \\
& \delta^{18} \mathrm{O}_{\mathrm{sa} / \mathrm{ref}}=\delta\left({ }^{18} \mathrm{O}^{18} \mathrm{O} /{ }^{18} \mathrm{O}\right)_{\mathrm{sa} / \mathrm{ref}}=\frac{\left(\frac{14 \mathrm{~N}^{18} \mathrm{O}_{2}{ }^{16} \mathrm{O}}{{ }^{14} \mathrm{~N}^{18} \mathrm{O}^{16} \mathrm{O}_{2}}\right)_{\text {ref }}=\Delta^{15} \mathrm{~N}^{18} \mathrm{O}_{\mathrm{sample}}}{\left(\frac{14 \mathrm{~N}^{18} \mathrm{O}_{2}{ }^{16} \mathrm{O}}{{ }^{14} \mathrm{~N}^{18} \mathrm{O}^{16} \mathrm{O}_{2}}\right)_{\text {reference }}}-1 \\
& \mid \text { if } \Delta^{18} \mathrm{O}^{18} \mathrm{O}_{\mathrm{ref}}=\Delta^{18} \mathrm{O}^{18} \mathrm{O}_{\text {sample }}
\end{aligned}
$$


$\delta^{17} O:$

$$
\begin{aligned}
& \delta^{17} \mathrm{O}_{\mathrm{sa} / \mathrm{ref}}=\frac{\left(\frac{{ }^{14} \mathrm{~N}^{17} \mathrm{O}^{16} \mathrm{O}_{2}}{{ }^{14} \mathrm{~N}^{16} \mathrm{O}_{3}}\right)_{\text {sample }}}{\left(\frac{{ }^{14} \mathrm{~N}^{17} \mathrm{O}^{16} \mathrm{O}_{2}}{{ }^{14} \mathrm{~N}^{16} \mathrm{O}_{3}}\right)_{\text {reference }}}-1 \\
& \delta^{17} \mathrm{O}_{\mathrm{sa} / \mathrm{ref}}=\delta\left({ }^{17} \mathrm{O} /{ }^{15} \mathrm{~N}\right)_{\mathrm{sa} / \mathrm{ref}}+\delta^{15} \mathrm{~N}_{\mathrm{sa} / \mathrm{ref}}+\delta\left({ }^{17} \mathrm{O} /{ }^{15} \mathrm{~N}\right)_{\mathrm{sa} / \mathrm{ref}} \cdot \delta^{15} \mathrm{~N}_{\mathrm{sa} / \mathrm{ref}} \\
& \text { with } \delta\left({ }^{17} \mathrm{O} /{ }^{15} \mathrm{~N}\right)_{\mathrm{sa} / \mathrm{ref}}=\frac{\left(\frac{14 \mathrm{~N}^{17} \mathrm{O}^{16} \mathrm{O}_{2}}{{ }^{15} \mathrm{~N}^{16} \mathrm{O}_{3}}\right)_{\text {sample }}}{\left(\frac{{ }^{14} \mathrm{~N}^{17} \mathrm{O}^{16} \mathrm{O}_{2}}{{ }^{15} \mathrm{~N}^{16} \mathrm{O}_{3}}\right)_{\text {reference }}}-1 \\
& \delta^{17} \mathrm{O}_{\mathrm{sa} / \mathrm{ref}}=\delta\left({ }^{17} \mathrm{O} /{ }^{18} \mathrm{O}\right)_{\mathrm{sa} / \mathrm{ref}}+\delta^{18} \mathrm{O}_{\mathrm{sa} / \mathrm{ref}}+\delta\left({ }^{17} \mathrm{O} /{ }^{18} \mathrm{O}\right)_{\mathrm{sa} / \mathrm{ref}} \cdot \delta^{18} \mathrm{O}_{\mathrm{sa} / \mathrm{ref}} \\
& \text { with } \delta\left({ }^{17} \mathrm{O} /{ }^{18} \mathrm{O}\right)_{\mathrm{sa} / \mathrm{ref}}=\frac{\left(\frac{{ }^{14} \mathrm{~N}^{17} \mathrm{O}^{16} \mathrm{O}_{2}}{{ }^{14} \mathrm{~N}^{18} \mathrm{O}^{16} \mathrm{O}_{2}}\right)_{\text {sample }}}{\left(\frac{14 \mathrm{~N}^{17} \mathrm{O}^{16} \mathrm{O}_{2}}{{ }^{14} \mathrm{~N}^{18} \mathrm{O}^{16} \mathrm{O}_{2}}\right)_{\text {reference }}}-1
\end{aligned}
$$

$\Delta^{17} O:$

$$
\begin{aligned}
& \Delta^{17} \mathrm{O}_{\mathrm{sa} / \mathrm{ref}}=\ln \left(\delta^{17} \mathrm{O}_{\mathrm{sa} / \mathrm{ref}}+1\right)-\lambda \cdot \ln \left(\delta^{18} \mathrm{O}_{\mathrm{sa} / \mathrm{ref}}+1\right) \\
& \Delta^{17} \mathrm{O}_{\mathrm{sa} / \mathrm{ref}}=\ln \left(\delta\left({ }^{17} \mathrm{O} /{ }^{18} \mathrm{O}\right)_{\mathrm{sa} / \mathrm{ref}}+1\right)+(1-\lambda) \cdot \ln \left(\delta^{18} \mathrm{O}_{\mathrm{sa} / \mathrm{ref}}+1\right) \\
& \Delta^{17} \mathrm{O}_{\mathrm{sa} / \mathrm{ref}}=(1-\lambda) \cdot \ln \left(\delta^{15} \mathrm{~N}_{\mathrm{sa} / \mathrm{ref}}+1\right)+\ln \left(\delta\left({ }^{17} \mathrm{O} /{ }^{15} \mathrm{~N}\right)_{\mathrm{sa} / \mathrm{ref}}+1\right)-\lambda \cdot \ln \left(\delta\left({ }^{18} \mathrm{O} /{ }^{15} \mathrm{~N}\right)_{\mathrm{sa} / \mathrm{ref}}+1\right)
\end{aligned}
$$

\section{Potential error from isotopologue ratio calculations}

Calculating isotope ratios from isotopologue abundances requires the assumption that all isotopologues are stochastically distributed. However, if the molecule exhibits clumping or anticlumping (i.e. non-stochastic) multiply substituted isotopologues, all other isotopoogues also have abundances that diverge slightly from a purely stochastic distribution. Due to the stoichiometry of the intramolecular isotope exchange reactions (below), deviations from a stochastic distribution result in the following linked changes for the two most abundant doubly substituted isotopologues of nitrate and their exchange reaction partners.

$$
\begin{aligned}
{ }^{15} \mathrm{~N}^{16} \mathrm{O}_{3}^{-}+{ }^{14} \mathrm{~N}^{18} \mathrm{O}^{16} \mathrm{O}_{2}^{-} & \leftrightarrow{ }^{15} \mathrm{~N}^{18} \mathrm{O}^{16} \mathrm{O}_{2}^{-}+{ }^{14} \mathrm{~N}^{16} \mathrm{O}_{3}^{-} \\
{ }^{14} \mathrm{~N}^{18} \mathrm{O}^{16} \mathrm{O}_{2}^{-}+{ }^{14} \mathrm{~N}^{18} \mathrm{O}^{16} \mathrm{O}_{2}^{-} & \leftrightarrow{ }^{14} \mathrm{~N}^{18} \mathrm{O}_{2}{ }^{16} \mathrm{O}^{-}+{ }^{14} \mathrm{~N}^{16} \mathrm{O}_{3}^{-}
\end{aligned}
$$

A $+x$ deviation in the abundance of ${ }^{15} \mathrm{~N}^{18} \mathrm{O}^{16} \mathrm{O}_{2}^{-}$necessarily coincides with a $+x$ deviation in the abundance of ${ }^{14} \mathrm{~N}^{16} \mathrm{O}_{3}^{-}$, and $\mathrm{a}-x$ deviation for both ${ }^{15} \mathrm{~N}^{16} \mathrm{O}_{3}^{-}$and ${ }^{14} \mathrm{~N}^{18} \mathrm{O}^{16} \mathrm{O}_{2}^{-}$.

Likewise, a $+y$ deviation in the abundance of ${ }^{14} \mathrm{~N}^{18} \mathrm{O}_{2}{ }^{16} \mathrm{O}^{-}$necessarily coincides with $\mathrm{a}+y$ deviation in the abundance of ${ }^{14} \mathrm{~N}^{16} \mathrm{O}_{3}^{-}$, and a $-2 y$ deviation for ${ }^{14} \mathrm{~N}^{18} \mathrm{O}^{16} \mathrm{O}_{2}^{-}$.

Taking these two clumped isotopologues into consideration, the resulting error in the stochastic assumption depends on $x$ and $y$ and can be expressed as:

$$
\begin{gathered}
\delta^{15} \mathrm{~N}_{\text {error }}=\frac{\frac{\left({ }^{15} \mathrm{~N}^{16} \mathrm{O}_{3}\right)_{\text {stochastic }}-x}{\left({ }^{14} \mathrm{~N}^{16} \mathrm{O}_{3}\right)_{\text {stochastic }}+x+y}}{\frac{\left(15 \mathrm{~N}^{16} \mathrm{O}_{3}\right)_{\text {stochastic }}}{\left({ }^{14} \mathrm{~N}^{16} \mathrm{O}_{3}\right)_{\text {stochastic }}}}-1 \\
\delta^{18} \mathrm{O}_{\text {error }}=\frac{\frac{\left({ }^{14} \mathrm{~N}^{18} \mathrm{O}^{16} \mathrm{O}_{2}\right)_{\text {stochastic }}-x-2 y}{\left({ }^{14} \mathrm{~N}^{16} \mathrm{O}_{3}\right)_{\text {stochastic }}+x+y}}{\frac{\left(14 \mathrm{~N}^{18} \mathrm{O}^{16} \mathrm{O}_{2}\right)_{\text {stochastic }}}{\left({ }^{14} \mathrm{~N}^{16} \mathrm{O}_{3}\right)_{\text {stochastic }}}}-1
\end{gathered}
$$


the $x$ and $y$ deviations from stochasticity are set by $\Delta^{15} \mathrm{~N}^{18} \mathrm{O}$ and $\Delta^{18} \mathrm{O}^{18} \mathrm{O}$ :

$$
\begin{aligned}
& \Delta^{15} \mathrm{~N}^{18} \mathrm{O}=\frac{\frac{\left({ }^{15} \mathrm{~N}^{18} \mathrm{O}^{16} \mathrm{O}_{2}\right)_{\text {measured }}}{\left({ }^{14} \mathrm{~N}^{16} \mathrm{O}_{3}\right)_{\text {measured }}}}{\frac{\left({ }^{15} \mathrm{~N}^{18} \mathrm{O}^{16} \mathrm{O}_{2}\right)_{\text {stochastic }}}{\left({ }^{14} \mathrm{~N}^{16} \mathrm{O}_{3}\right)_{\text {stochastic }}}}-1=\frac{\frac{\left({ }^{15} \mathrm{~N}^{18} \mathrm{O}^{16} \mathrm{O}_{2}\right)_{\text {stochastic }}+x}{\left({ }^{14} \mathrm{~N}^{16} \mathrm{O}_{3}\right)_{\text {stochastic }}+x+y}}{\frac{\left(15 \mathrm{~N}^{18} \mathrm{O}^{16} \mathrm{O}_{2}\right)_{\text {stochastic }}}{\left({ }^{14} \mathrm{~N}^{16} \mathrm{O}_{3}\right)_{\text {stochastic }}}}-1 \\
& \Delta^{18} \mathrm{O}^{18} \mathrm{O}=\frac{\frac{\left({ }^{14} \mathrm{~N}^{18} \mathrm{O}_{2}{ }^{16} \mathrm{O}\right)_{\text {measured }}}{\left({ }^{14} \mathrm{~N}^{16} \mathrm{O}_{3}\right)_{\text {measured }}}}{\frac{\left(14 \mathrm{~N}^{18} \mathrm{O}_{2} \mathrm{O}^{16}\right)_{\text {stochastic }}}{\left({ }^{14} \mathrm{~N}^{16} \mathrm{O}_{3}\right)_{\text {stochastic }}}}-1=\frac{\frac{\left({ }^{14} \mathrm{~N}^{18} \mathrm{O}_{2}{ }^{16} \mathrm{O}\right)_{\text {stochastic }}+y}{\left({ }^{14} \mathrm{~N}^{16} \mathrm{O}_{3}\right)_{\text {stochastic }}+x+y}}{\frac{\left(14 \mathrm{~N}^{18} \mathrm{O}_{2}{ }^{16} \mathrm{O}\right)_{\text {stochastic }}}{\left({ }^{14} \mathrm{~N}^{16} \mathrm{O}_{3}\right)_{\text {stochastic }}}}-1
\end{aligned}
$$

Using $\Delta^{15} \mathrm{~N}^{18} \mathrm{O}$ and $\Delta^{18} \mathrm{O}^{18} \mathrm{O}$ to eliminate $x$ and $y$ from the equations for $\delta^{15} \mathrm{~N}_{\text {error }}$ and $\delta^{18} \mathrm{O}_{\text {error }}$ yields:

$$
\begin{aligned}
& \delta^{15} \mathrm{~N}_{\text {err }}=- \\
& \frac{\Delta{ }^{15} \mathrm{~N}^{18} \mathrm{O} \cdot{ }^{15} \mathrm{~N}^{18} \mathrm{O}^{16} \mathrm{O}_{2} \cdot\left({ }^{14} \mathrm{~N}^{16} \mathrm{O}_{3}+{ }^{15} \mathrm{~N}^{16} \mathrm{O}_{3}-{ }^{14} \mathrm{~N}^{18} \mathrm{O}_{2}{ }^{16} \mathrm{O}\right)+\Delta{ }^{18} \mathrm{O}^{18} \mathrm{O} \cdot{ }^{14} \mathrm{~N}^{18} \mathrm{O}_{2}{ }^{16} \mathrm{O} \cdot\left({ }^{15} \mathrm{~N}^{16} \mathrm{O}_{3}+{ }^{15} \mathrm{~N}^{18} \mathrm{O}^{16} \mathrm{O}_{2}\right)}{{ }^{15} \mathrm{~N}^{16} \mathrm{O}_{3} \cdot\left({ }^{14} \mathrm{~N}^{16} \mathrm{O}_{3}-{ }^{15} \mathrm{~N}^{18} \mathrm{O}^{16} \mathrm{O}_{2}-{ }^{14} \mathrm{~N}^{18} \mathrm{O}_{2}{ }^{16} \mathrm{O}\right)}
\end{aligned}
$$

$$
\begin{aligned}
& \delta^{18} \mathrm{O}_{\text {err }}=- \\
& \frac{\Delta^{15} \mathrm{~N}^{18} \mathrm{O} \cdot{ }^{15} \mathrm{~N}^{18} \mathrm{O}^{16} \mathrm{O}_{2}\left({ }^{14} \mathrm{~N}^{16} \mathrm{O}_{3}+{ }^{14} \mathrm{~N}^{18} \mathrm{O}^{16} \mathrm{O}_{2}+{ }^{14} \mathrm{~N}^{18} \mathrm{O}_{2}{ }^{16} \mathrm{O}\right)+\Delta \Delta^{18} \mathrm{O}^{18} \mathrm{O} \cdot{ }^{14} \mathrm{~N}^{18} \mathrm{O}_{2}{ }^{16} \mathrm{O}\left(2^{14} \mathrm{~N}^{16} \mathrm{O}_{3}+{ }^{14} \mathrm{~N}^{18} \mathrm{O}^{16} \mathrm{O}_{2}-{ }^{15} \mathrm{~N}^{18} \mathrm{O}^{16}(\right.}{{ }^{14} \mathrm{~N}^{18} \mathrm{O}^{16} \mathrm{O}_{2} \cdot\left({ }^{14} \mathrm{~N}^{16} \mathrm{O}_{3}-{ }^{15} \mathrm{~N}^{18} \mathrm{O}^{16} \mathrm{O}_{2}-{ }^{14} \mathrm{~N}^{18} \mathrm{O}_{2}{ }^{16} \mathrm{O}\right)}
\end{aligned}
$$

\section{Error visualization}

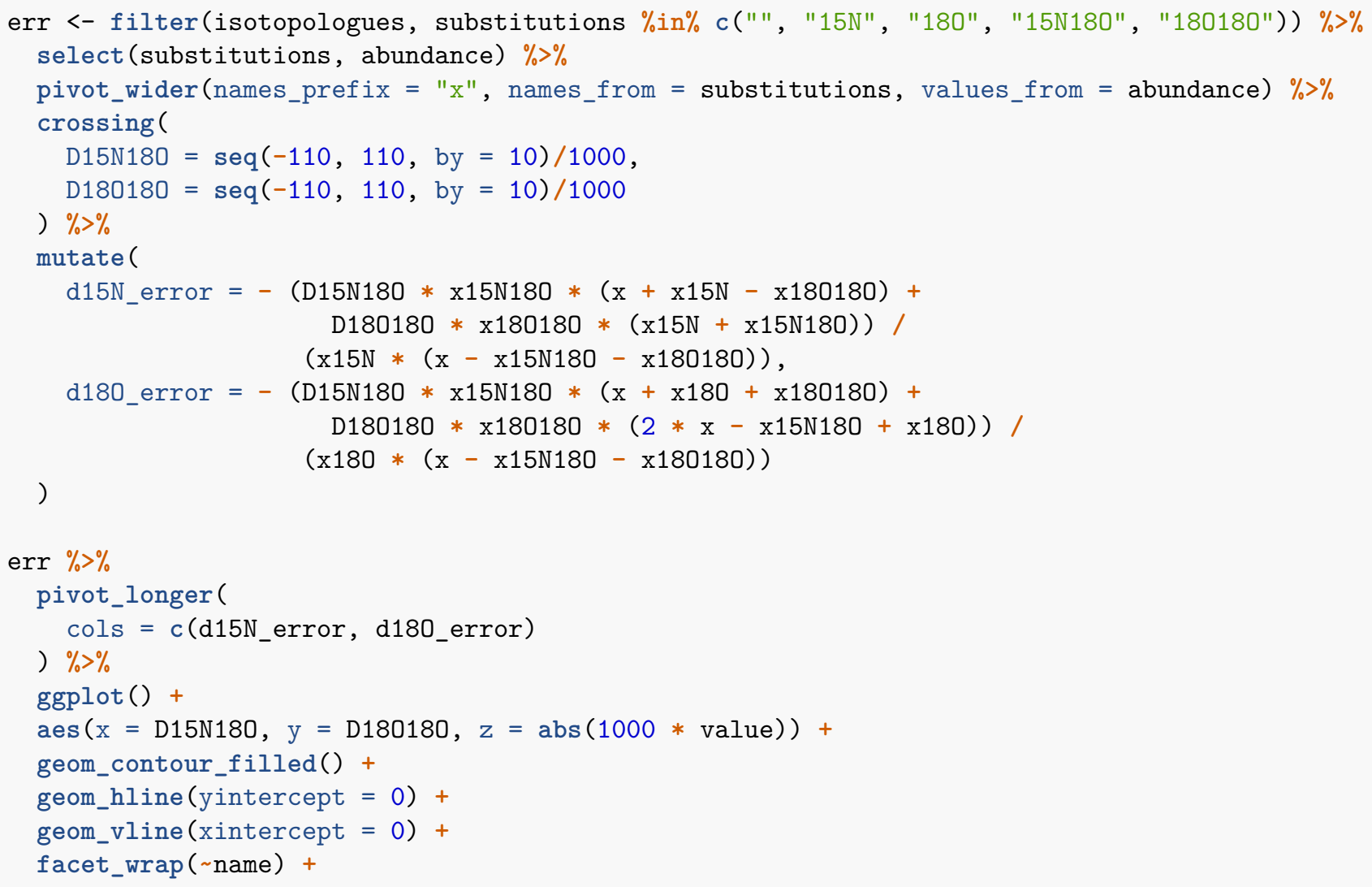




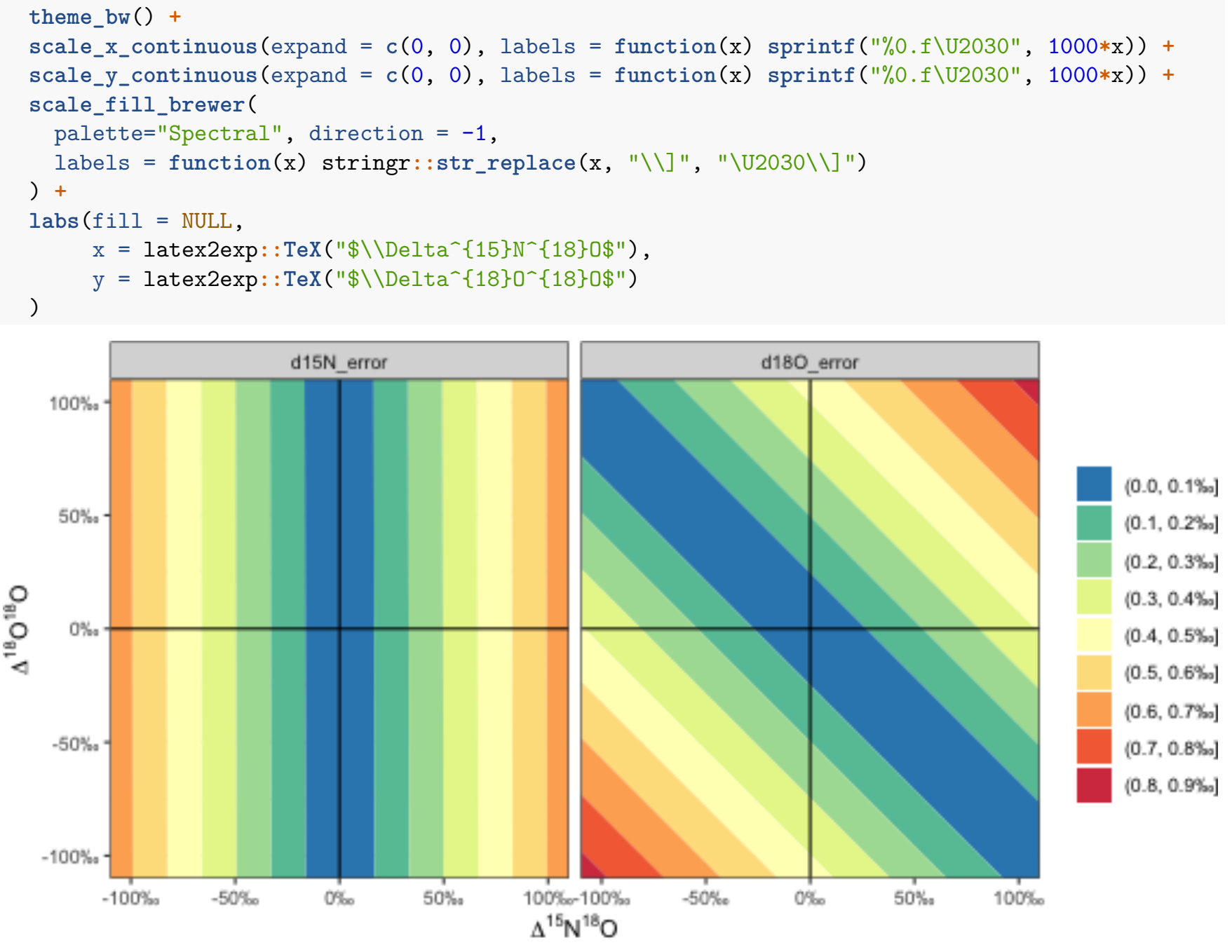

